\title{
Discovery of a new branch of the Taurid meteoroid stream as a real source of potentially hazardous bodies
}

\author{
P. Spurný ${ }^{1}$, J. Borovička ${ }^{1}$, H. Mucke ${ }^{2}$, and J. Svoreň ${ }^{3}$ \\ 1 Astronomical Institute of the Czech Academy of Sciences, 25165 Ondřejov, Czech Republic \\ e-mail: pavel.spurny@asu.cas.cz \\ 2 Astronomisches Büro, 1230 Wien, Austria \\ 3 Astronomical Institute of the Slovak Academy of Sciences, 05960 Tatranská Lomnica, Slovak Republic
}

Received 14 March 2017 / Accepted 1 May 2017

\begin{abstract}
Taurid meteor shower produces prolonged but usually low activity every October and November. In some years, however, the activity is significantly enhanced. Previous studies based on long-term activity statistics concluded that the enhancement is caused by a swarm of meteoroids locked in 7:2 resonance with Jupiter. Here we present precise data on 144 Taurid fireballs observed by new digital cameras of the European Fireball Network in the enhanced activity year 2015. Orbits of 113 fireballs show common characteristics and form together a well defined orbital structure, which we call new branch and which was evidently responsible for the enhanced activity. This new branch is part of Southern Taurids and was encountered by the Earth between October 25 and November 17. We found that this branch is characterized by longitudes of perihelia lying between $155.9-160^{\circ}$ and latitudes of perihelia between 4.2-5. $7^{\circ}$. Semimajor axes are between 2.23-2.28 AU and indeed overlap with the 7:2 resonance. Eccentricities are in wide range $0.80-0.90$. The most eccentric orbits with lowest perihelion distances were encountered at the beginning of the activity period. The orbits form a concentric ring in the inner solar system. The masses of the observed meteoroids were in a wide range from $0.1 \mathrm{~g}$ to more than $1000 \mathrm{~kg}$. We found that all meteoroids larger than $300 \mathrm{~g}$ were very fragile (type IIIB), while those smaller than $30 \mathrm{~g}$ were much more compact (mostly of type II and some of them even type I). Based on orbital characteristics, we argue that asteroids 2015 TX24 and 2005 UR, both of diameters 200-300 m, are direct members of the new branch. It is therefore very likely that the new branch contains also numerous still not discovered objects of decameter or even larger size. Since asteroids of sizes of tens to hundreds meters pose a threat to the ground even if they are intrinsically weak, impact hazard increases significantly when the Earth encounters the Taurid new branch every few years. Further studies leading to better description of this real source of potentially hazardous objects, which can be large enough to cause significant regional or even continental damage on the Earth, are therefore extremely important.
\end{abstract}

Key words. meteorites, meteors, meteoroids - comets: individual: 2P/Encke - minor planets, asteroids: general - Earth

\section{Introduction}

The Taurid meteoroid stream is one of the most studied meteoroid streams. This stream produces at least four meteor showers on Earth: the Northern and Southern Taurids, both active from end of September until December; the Daytime $\zeta$-Perseids, active from end of May to the beginning of July; and the Daytime $\beta$-Taurids, active in June and the first half of July (Jenniskens 2006). Other showers may be also related to the Taurid stream, namely the Piscids in September, $\chi$-Orionids in December, and Daytime May Arietids in May (Jenniskens 2006). Since the work of Whipple (1940), the short period comet 2P/Encke has been considered the most probable parent body of the Taurid stream. It was, nevertheless, proposed that $2 \mathrm{P} /$ Encke is just a fragment of a much larger comet, which was disrupted $10^{3}-$ $10^{4} \mathrm{yr}$ ago and formed the whole Taurid complex including a number of asteroids (Clube \& Napier 1984; Napier 2010). As more and more asteroids were being discovered over time, the number of asteroids proposed by various authors as members of the Taurid complex increased (e.g., Asher et al. 1993; Babadzhanov 2001; Porubčan et al. 2006; Babadzhanov et al. 2008; Olech et al. 2016). The problem is that the Taurid stream is very extended and the low-inclination short-period Taurid orbits are very common for near-Earth asteroids. Many proposed associations can be therefore just random coincidences. Indeed, the spectra of six large asteroids proposed as members of the Taurid complex showed that five of them are inconsistent with a cometary origin (Popescu et al. 2014).

The activity of Taurids is prolonged but usually of low level. In some years, however, the activity is enhanced, especially in terms of large numbers of bright meteors (fireballs). Asher \& Clube (1993) proposed that there is a resonant swarm of meteoroids trapped in the 7:2 resonance with Jupiter. The expected extent of the swarm was $\pm 30-40^{\circ}$ in mean anomaly. Asher \& Izumi (1998) showed that enhanced Taurid activity indeed occurred in the years when the center of the swarm was less than $40^{\circ}$ in mean anomaly from the Earth at the beginning of November (the date of Taurid maximum). Asher \& Clube (1993) predicted that future encounters would occur in 1995, 1998, 2005, and 2008. In 1995, enhanced Taurid activity was observed by the European Fireball Network (EN) when the rate of registered Taurid fireballs was noticeably higher than is usual for the EN at that time of year (Spurný 1996). Apart from regular Southern and Northern Taurids, five fireballs observed during the last week of October 1995 had distinct but very similar orbits. The radiants lay near the regular southern Taurid radiant, but the initial velocities were larger $\left(V_{\mathrm{g}}=33.1 \pm 0.3 \mathrm{~km} \mathrm{~s}^{-1}\right)$. As a result these orbits had significantly larger semimajor axes 


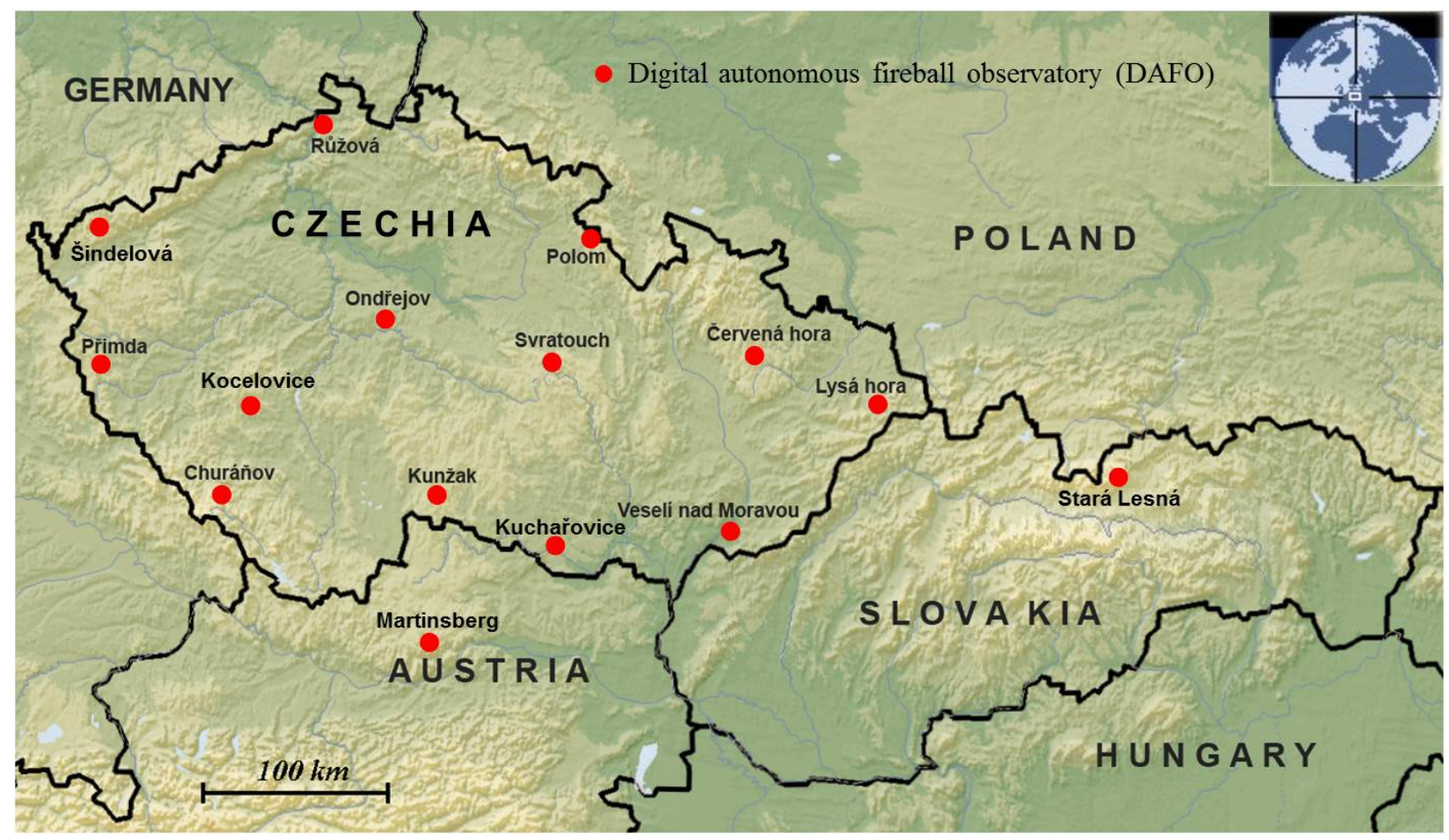

Fig. 1. Stations of the fireball network located in Czech Republic, Slovakia and Austria where DAFO are placed (status November 2015).

$(a=2.52 \pm 0.08 \mathrm{AU})$, eccentricities $(e=0.905 \pm 0.004)$ and inclinations $\left(i=6.2^{\circ} \pm 0.4^{\circ}\right)$, and smaller perihelion distances $(q=0.241 \pm 0.009 \mathrm{AU})$ than the regular Southern Taurid orbit. The existence of this well-defined cluster of similar Taurid meteoroids very probably means that the enhanced activity in 1995 was caused by a new relatively compact subsystem of the Taurid complex close to the Southern Taurids. As mentioned in Jenniskens (2006), this observation identifies, for the first time, a meteor outburst associated with the Taurid shower, and by implication the Earth crossing a relatively young dust trail. The 1995 enhanced activity was also confirmed by visual observations (Dubietis \& Arlt 2007), both in terms of increased overall activity and increased percentage of fireballs in the period from October 23 to November 15 (McBeath 1999). Similarly Johannink \& Miskotte (2006), also from visual observations, confirmed increased Taurid activity in 1998 and 2005 and suggested that the Southern Taurids are responsible for the higher activity in resonance years.

Shiba (2016) analyzed Taurid video observations from 2007 to 2015, including swarm encounter years 2008, 2012, and 2015 (see the webpage of $\mathrm{D}$. Asher ${ }^{1}$ for swarm encounter predictions). He confirmed that the enhanced activity is exclusively due to Southern Taurids. Shiba also studied the dependency of orbital elements on time and found that not only the mean orbital period of swarm meteoroids but also that of Northern Taurids correspond to the 7:2 resonance with Jupiter. The eccentricity was found to decrease and perihelion distance to increase with time.

The work of Shiba work is statistical in nature, involving thousands of meteors but with large individual uncertainties. Here we present precise data on 144 Taurid fireballs observed by new digital cameras of the EN in 2015. The description of the observational system, examples of the data, and demonstration of their precision are given in Sects. 2 and 3. In Sect. 4 we show that the enhanced activity in 2015 was caused by a well-defined

\footnotetext{
1 http://star.arm.ac.uk/ dja/taurid/swarmyears.html
}

branch of Taurid meteoroids. We concentrate our study on orbital elements and only briefly discuss the physical properties of the meteoroids. In Sect. 5 we show that several known asteroids also belong to the branch, which caused the 2015 activity. The implications of our work are discussed in Sect. 6.

\section{Observational techniques and data acquisition}

The data reported here were obtained by the EN. The core of the network, located in the Czech Republic, has been modernized several times (Spurný et al. 2007). But the last significant improvement has been realized during the last three years when a completely new instrument, the high-resolution digital autonomous fireball observatory (DAFO), was developed and gradually installed on the stations of the fireball network between November 2013 and September 2015. These new all-sky digital cameras are working alongside the older analog (using photographic films) autonomous all-sky cameras (AFO) on the majority of Czech stations but this older system based on AFOs is gradually being decommissioned. At the end of 2015 the DAFOs were installed on 13 stations around the Czech Republic (Šindelová and Kocelovice stations are completely new and were built in mid-2015). Apart from the Czech territory, two DAFOs were installed on the already working stations in Slovakia and Austria, respectively. The first DAFO was installed at the observatory of the Slovak Academy of Sciences in Tatranská Lomnica, where one AFO also remains in full operation, and the second, installed at the Waldviertel Observatory in Martinsberg (Austria), substituted the previous AFO system in September 2015. This core of the EN as schematically shown in Fig. 1 also cooperates with other parts and systems located in neighboring European countries but data used in this study are solely acquired by the stations based on the DAFO (vast majority of used records) and AFO cameras as described above. 
The imaging part of the DAFO system is comprised of a full frame Canon 6D digital camera and a Sigma fish-eye lens $(8 \mathrm{~mm}$ $f / 3.5)$ equipped with an electronic LCD shutter for speed determination. In standard regime 16 interruptions and $35 \mathrm{~s}$ long exposure are used. To avoid possible loss of data during reading time of the CMOS sensor, we use two identical imaging sets, which work in alternation mode with $5 \mathrm{~s}$ overlap. The older AFOs analog imaging part is comprised of a Zeiss Distagon fisheye lens ( $30 \mathrm{~mm} f / 3.5$ ). Large format panchromatic sheet films $(9 \times 12 \mathrm{~cm}$, Ilford FP4) are used. The diameter of the sky on the image is $8 \mathrm{~cm}$ and usually one exposure is taken per night. Mechanical shutter with 15 interruptions per second is used. The sensitivity limit is -4 mag for AFO (about 2-3 mag lower around the full Moon period) and -2 mag for DAFO (with lower dependence on lunar phase). Apart from the imaging part, each DAFO and AFO is equipped with an all-sky radiometer with time resolution of 5000 samples per second and with similar sensitivity limit (in the moonless nights) like the imaging system but with much higher dynamic range. These radiometers serve several purposes, such as the real-time detection of fireballs, their exact absolute timing (system time is continuously corrected by the PPS pulse of the GPS), recording of detailed light curve profiles, and for precise photometry, especially for brighter events when digital images become saturated as shown in one example later.

The data presented in this study were obtained almost completely by the new digital autonomous system (DAFO). Thanks to their higher sensitivity, fireball observations from DAFO contain more information especially in the beginning and terminal parts of the luminous trajectory in comparison with AFO. Another important advantage of DAFO is the ability to work during periods when it is not completely dark (twilight periods) and not completely clear (partly cloudy sky) as well. The data from the new digital system allow us to reliably determine all basic parameters of sufficiently bright fireballs up to the distance of $300 \mathrm{~km}$ from the stations (for special cases even up to $600 \mathrm{~km}$ ). It means that with the current number and displacement of stations (see Fig. 1) we effectively cover territory of roughly 3 million square kilometers at least, i.e., a large part of Central Europe. All the advantages mentioned above significantly increased the efficiency of our observations; and in direct comparison with the efficiency of the previous analog AFO system the number of recorded fireballs increased at least three times. When we combine this increased efficiency with improved analysis techniques, which we developed and gradually improved especially in the last several years, we obtain results than were not reached by any previous observing system used within the EN.

\section{Data reduction}

As described above, our fully automated instruments DAFO and AFO provide us with two kinds of data: all-sky photographic records and high-resolution radiometric light curves. For the complete analysis of every fireball that was recorded from at least two stations (the vast majority of the presented fireballs were recorded from more than two stations) we use our own procedures, methods and analysis software. All-sky images taken in the raw format are measured by the FishScan application, which allows semiautomatic measurement of positions, speed, and photometry. Usually the photometry from digital images is reliable up to -8 apparent magnitude, brighter events start to be saturated after reaching this brightness. However, thanks to the high dynamic range of radiometers, which are incorporated in each DAFO and AFO, we are able to obtain precise photometry also for much brighter fireballs, even for superbolides as will be shown later. We can calibrate radiometric records using not saturated parts of the light curve obtained from photographic records. Our whole procedure is demonstrated on the example below.

The Taurid fireball of November 5, 2015, 23:12:01 UT, was recorded photographically and photoelectrically at seven stations in our network. For a complete analysis of this fireball, we chose records taken from four stations that were close to its atmospheric trajectory and were sufficient for reliable determination of all parameters describing atmospheric trajectory, dynamics, photometry, and heliocentric orbit of this fireball. A selection of all-sky images of the fireball taken at individual stations are shown in Fig. 2.

The first step after measurement of all four digital images and their astrometric reduction is computation of the atmospheric luminous trajectory. We use two different methods described in Ceplecha (1987): the so-called plane method, and in Borovička (1990), the so-called least-squares method. A first independent check of the results is that the values describing the atmospheric trajectory obtained from these two methods turn out to be the same within the uncertainties. Lateral deviations of all measured points from the resulting atmospheric trajectory (zero line) are shown in Fig. 3. This plot illustrates the high reliability of the astrometric solution. The spread of the measured points from individual stations is random and the standard deviation is only $7 \mathrm{~m}$. In this context it is also important to mention how far each station (camera) was from the fireball. Exact distances of the beginning and terminal points $R_{(B \div E)}$ for each station were as follows:

$R_{(B \div E)}(107)=141.8 \div 92.7 \mathrm{~km}$
$R_{(B \div E)}(126)=149.1 \div 119.5 \mathrm{~km} ;$
$R_{(B \div E)}(102)=176.6 \div 143.3 \mathrm{~km} ;$
$R_{(B \div E)}(114)=221.5 \div 189.8 \mathrm{~km}$.

This example nicely illustrates that our records and methods provide us with a precision of the atmospheric trajectory determination of about $10 \mathrm{~m}$ for fireballs that are still about $200 \mathrm{~km}$ away the stations. Most of the Taurids in this study, especially the fainter ones, were below or around this distance, and only several of the brightest cases were at much larger distances from the stations. The most distant Taurid was the superbolide EN311015_180520, which was also recorded by the cameras at distances of up to $630 \mathrm{~km}$ (stations that were used for analysis). Data precision for such a distant and difficult to measure case is about $140 \mathrm{~m}$, which is still good. The precision is crucial not only for the determination of the position of the trajectory in the atmosphere, but also for the determination of the direction of flight of the meteoroid, in other words, the position of the apparent radiant, which is important for determination of the heliocentric orbit of the meteoroid. The coordinates of the apparent radiant for this particular fireball were

$\alpha_{\text {app }}=54.947^{\circ} \pm 0.007^{\circ}, \delta_{\text {app }}=16.196^{\circ} \pm 0.004^{\circ}$.

When computing the local azimuth and slope of the trajectory, we took into account the curvature of bolide trajectory due to gravity, which can be significant for longer fireballs with very precise data. For the EN051115_231201 it is only $0.02^{\circ}$.

The second step is the determination of the velocity of the fireball. The data in this study are so good that it enabled us 


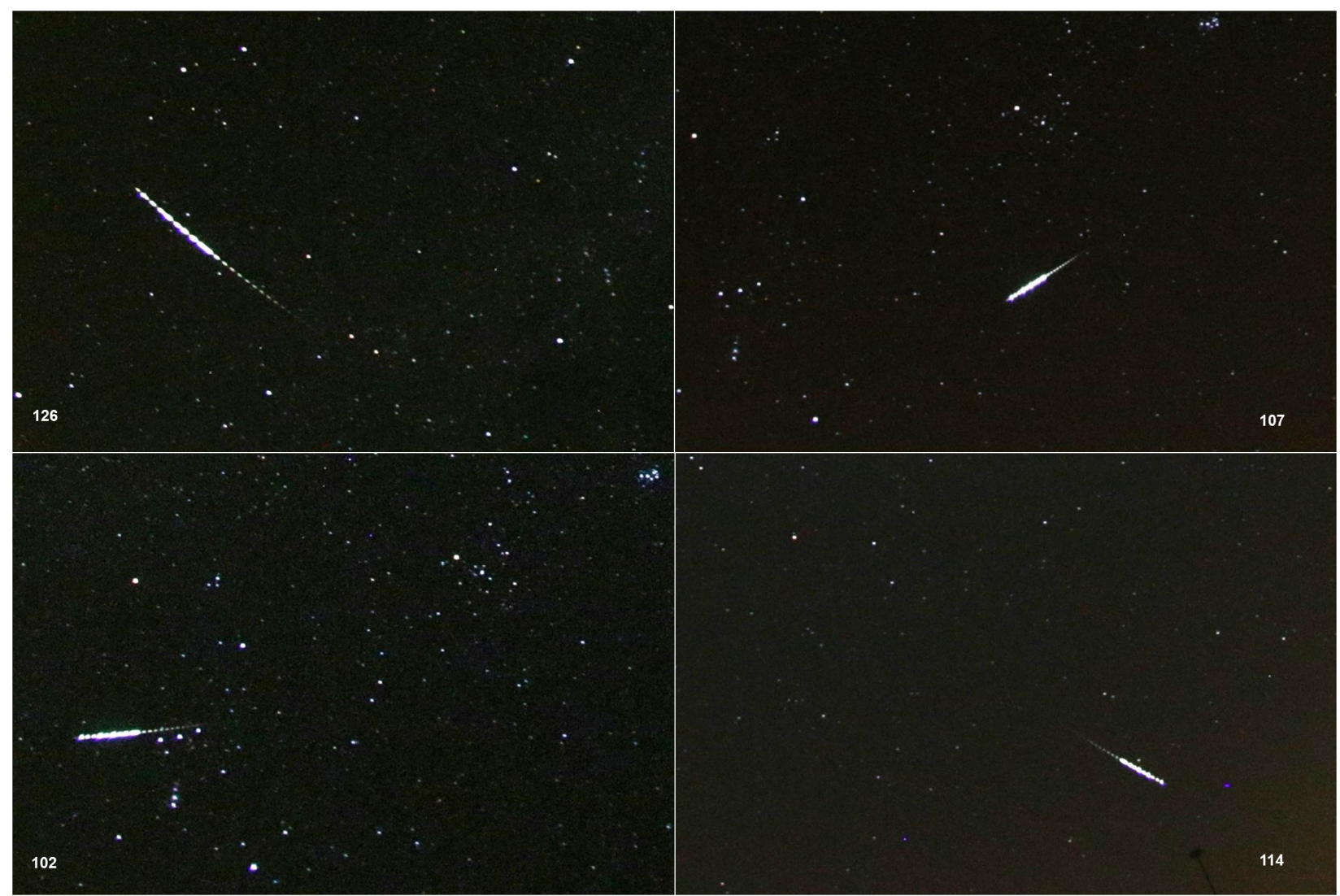

Fig. 2. Detailed views of the EN051115_231201 Taurid fireball recorded by DAFOs at the stations 126 Martinsberg, 102 Kunžak, 107 Kuchařovice, and 114 Červená hora. All-sky images from these stations were used for the analysis.

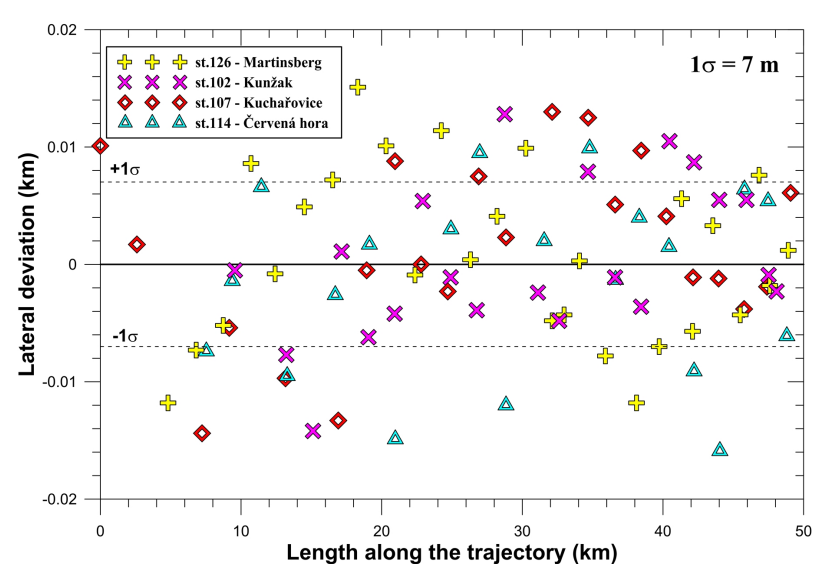

Fig. 3. Lateral deviations of all measured points on the fireball luminous path from the available records. The $Y$-axis scale is highly enlarged and one standard deviation for any point on the fireball trajectory is only $7 \mathrm{~m}$.

to use the method described in Ceplecha et al. (1993) for the vast majority of fireballs. Successful application of this model is very sensitive to the quality of the lengths for each individual measured velocity point corresponding to a single shutter break. This rigorous physical model provides the speed at any point on the trajectory but for the presented study, which is focused on the orbital analysis, the initial velocity is the most important. For the sample fireball we obtained a four parameter (non-fragmenting) solution including initial velocity for each station and all solutions were very similar. Nevertheless for the final dynamic solution and initial velocity determination, not only for this particular case but for all cases in this study, we used a slightly different approach. We put all measured shutter breaks from all used images together (timescales on all DAFOs are correlated) and we applied the Ceplecha method on this unified data set. This approach significantly increases reliability of the resulted dynamic solution. It is useful especially for shorter fireballs such as Taurids because they are moderately fast meteoroids of cometary origin (i.e., relatively fragile) and the number of measured breaks on one image can be limited. Therefore, every independent measurement can be very useful in obtaining a reliable value of the initial velocity. Moreover, for some fireballs the non-fragmenting solution applied to the whole trajectory was not adequate and we had to omit the terminal part of the fireball to obtain a realistic value of the initial velocity. This was also the case of the sample Taurid fireball EN051115_231201 as can be seen for example in Fig. 4 where several bright flares corresponding to fragmentation events are clearly visible. The resulting value of initial velocity of the EN051115_231201 Taurid fireball is $31.221 \pm 0.037 \mathrm{~km} \mathrm{~s}^{-1}$.

The next step in the analysis of the available records is the exact photometry of the fireball. We have two different data types, those from photographic records and radiometers, from which we can determine the brightness of the fireball and its initial mass based on photometry. As mentioned above, we measure digital images in 14-bit raw format. We found that this limited dynamic range of the used CMOS sensor is sufficient for fireballs with apparent magnitude up to about -8 . Above this limit the measured signal starts to be saturated. As shown in Table A.2, which contains basic physical data of the presented Taurids, this method 


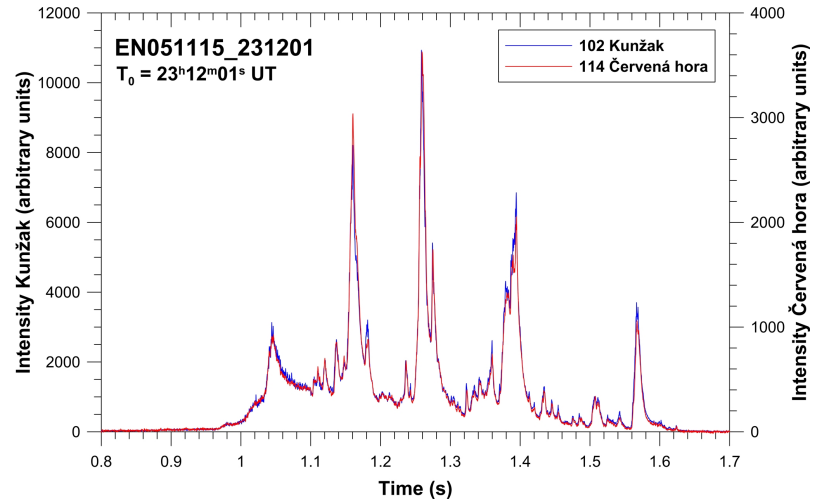

Fig. 4. Radiometric light curves of the EN051115_231201 fireball taken by fast photometers (5000 samples/s) at Kunžak (blue) and Červená hora (red) stations. These apparent (not corrected for distance) light curves taken from places $185 \mathrm{~km}$ apart demonstrate perfect compliance of both records; small differences in heights of individual peaks are caused by different distances to the fireball.

can be reliably used for about $75 \%$ of all cases. The remaining $25 \%$ of presented cases are such bright fireballs that their digital images are partly or even almost completely saturated. For such fireballs we have different methods to describe their brightness. One solution to this problem is the use of simultaneous photographic images taken by the AFO on the film. The response of the film emulsion is logarithmic, which means that the photographic film has much higher dynamic range; we use Ilford FP4 panchromatic films with sensitivity 125 ASA. This is a quite straightforward method and we used it in few cases, but a still much more appropriate and accurate way is the use of the light curves taken by the radiometers, which are in our cameras and still have much higher dynamic range. The apparent (i.e., not corrected for distance) high-resolution (5000 samples/s) radiometric light curves of the EN051115_231201 fireball taken by radiometers at Kunžak (blue) and Červená hora (red) stations are shown in Fig. 4. The close agreement between the different records, a testament to the high precision of the data, is evident. As for other two closer stations to the fireball, the radiometric light curves have exactly the same profile and could be used for fireball photometry. However, we cannot use radiometric light curves directly because individual radiometers have different sensitivity and are not calibrated to obtain absolute photometry. For the purpose of calibrating these records we combine photometry from both methods. We measure the meteor signal on the digital image and for calibration we use that part of the photographic image, which is not saturated and at the same time well above the noise of the measured signal from both the photographic records and corresponding radiometric records. This is usually somewhere in the interval between -4 and -7 mag. However, we have to relate the timescale of the photograph to the absolute timescale of the radiometer. For this purpose we use time marks (breaks of double length) made by the electronic shutter along the luminous path of the recorded fireball on the beginning of each second. This defines the exact absolute time of this measured point, which can be simply identified with the corresponding point on the radiometric light curve. Both radiometer and electronic shutter are continuously corrected by the PPS pulse of the GPS so the absolute timing of both records is given with high precision.

The result of this procedure is illustrated in Fig. 5 in which photographic and radiometric light curves from all used stations in absolute magnitudes are plotted. The first evident result is that,

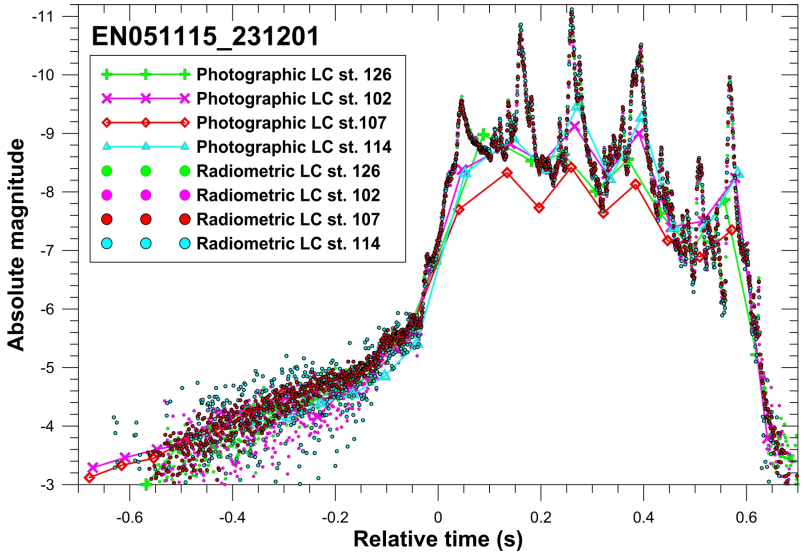

Fig. 5. Photographic and radiometric light curves of the EN051115_231201 fireball in absolute magnitudes.

especially for shorter and faster fireballs containing bright and short significant flares, the photographic photometry cannot correctly describe the shape of the light curve because of the low time resolution. The electronic shutter, on the other hand, has a resolution of 16 interruptions per second with the same length for the on/off state. This means that blind time lasts exactly $0.03125 \mathrm{~s}$ and it is sometimes longer than the duration of a flare or at least its brightest part. As can be seen in Figs. 4 and 5, this is exactly the case of fireball EN051115_231201, where most of the light is contained within five distinct and quite short flares that are only partly recorded (or even missing) on the image. Another aspect that is evident in Fig. 5 is the saturation of the photographic records. The absolute photographic photometry profile (brightest parts of the light curves) is, unlike the radiometric photometry, quite different for individual stations. However it confirms the saturation effect because stations, which are more distant from the fireball give higher absolute maximum brightness. It means that these records are not as saturated as the records from closer stations. The last aspect, which is worth mentioning in connection with the photometry shown in Fig. 5, is that for correct calibration of radiometric light curves it is much better to use the radiometric record and photographic image from the closest station where the signal-to-noise ratio is the most favorable. This is valid especially for the fireballs as in the case described here, when the increase of the brightness is very steep and the suitable (not saturated) interval of magnitudes is very short.

A general conclusion is that the high-resolution radiometric records are crucial for correct recovery of the photometry of all brighter fireballs, especially those that contain distinct flares. We note that the photometry based on radiometric light curves was determined for about $90 \%$ of analyzed Taurids in this study.

As explained above, apart from the precise photometry of the recorded fireballs, radiometric records provide us with a very accurate absolute time of each event. This important parameter is, along with the initial velocity and radiant position, necessary for reliable determination of the heliocentric orbit of the observed fireball. The orbits were computed by the method of Ceplecha (1987).

To compute the photometric mass of the meteoroid, the velocity dependence of the luminous efficiency was taken from ReVelle \& Ceplecha (2001). The mass dependence was ignored by substituting $10 \mathrm{~kg}$ for the mass in their formula. Specifically, the luminous efficiency for velocities above $25.4 \mathrm{~km} \mathrm{~s}^{-1}$ was assumed to be directly proportional to the velocity, reaching $6.5 \%$ 


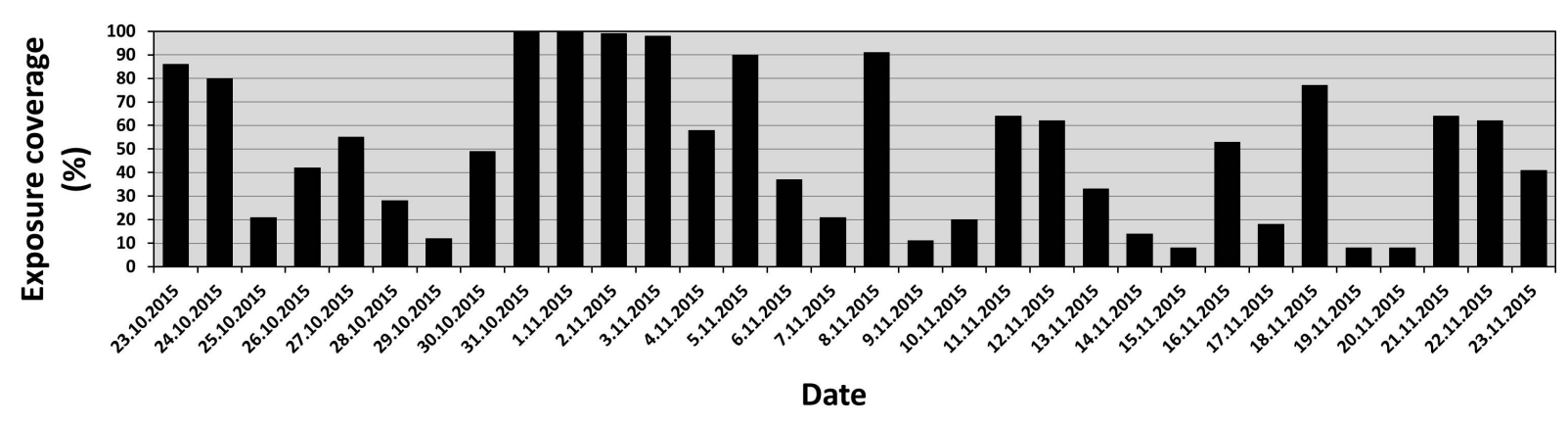

Fig. 6. Exposure coverage representing observing conditions in Central Europe during activity of Taurids in 2015. It is the fraction of real time when all cameras in the network exposed to their total prescribed exposure time. The date corresponds to the evening date of the whole night.

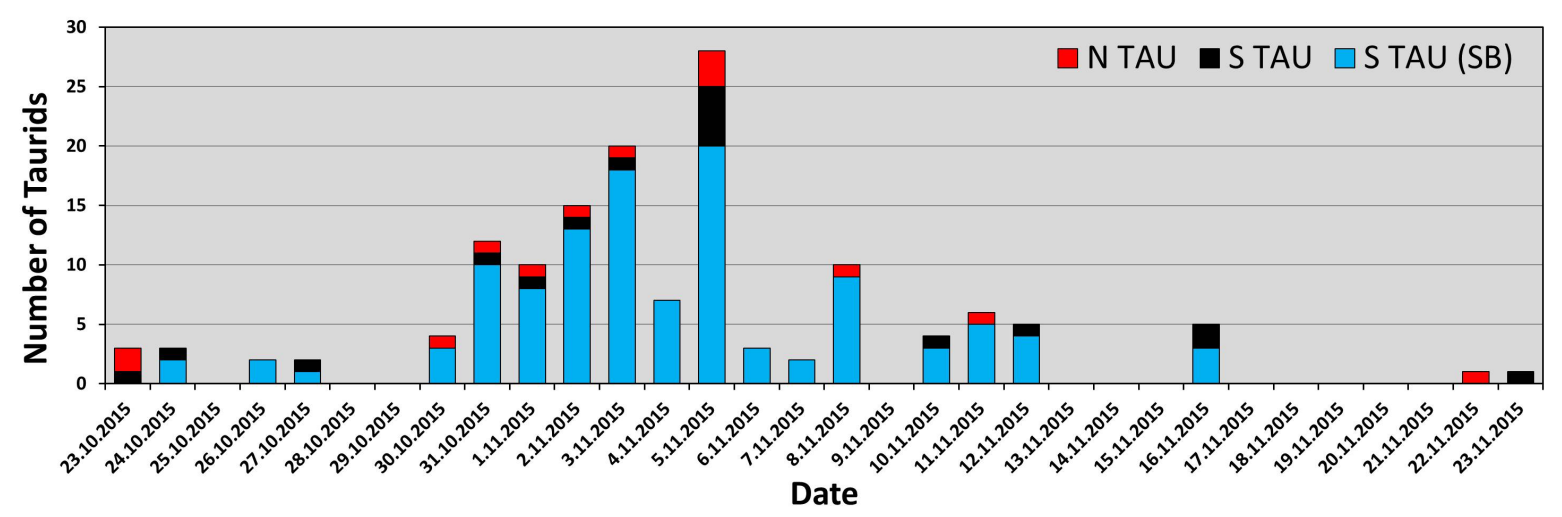

Fig. 7. Activity of Taurids recorded by the DAFO cameras of the European Fireball Network in 2015. The number of presented Taurid fireballs in the plot is 143 (total number is 144); S TAU from 28.11.2015 is out of range of the date axis. Date corresponds to the evening date of the whole night.

at $30 \mathrm{~km} \mathrm{~s}^{-1}$. A factor of $1500 \mathrm{~W}$ for zero magnitude meteor (Ceplecha et al. 1998) was used to convert magnitudes into bolometrically radiated energy.

The above-mentioned example clearly demonstrates high precision and reliability of all parameters describing the atmospheric trajectory, dynamics, photometry, and heliocentric orbit not only for this particular case, but also for all Taurid fireballs presented in Tables A.1 and A.2. For this complex analysis of all presented Taurid fireballs, i.e., the astrometric reduction of the images, atmospheric trajectory computation, dynamic and photometric solutions, and finally orbital calculations, we used our new software package BOLTRACK (J. Borovička).

Although the autumn weather, especially in November, is notoriously cloudy in Central Europe, the year 2015 was not so bad. There were several clear nights, especially in the beginning of November, and only a few nights were completely cloudy practically at all stations. This situation is illustrated in Fig. 6 in which the ratio of real to prescribed exposure time for all stations in the network altogether and for each individual night covering the Taurids activity in 2015 is shown. Since the DAFOs also work when the sky is only partly clear, some fireballs were captured even in the nights when it was mostly cloudy and could be still used for this study. On the other hand, some fireballs were recorded only from one station or their records were of a quality that is insufficient to merit scientific analysis. Such cases were excluded from our study. As a result of relatively favorable weather conditions and the capability of our network, we were able to cover the whole period of the enhanced Taurid activity from the last decade of October to mid-November as shown in Fig. 7. It is difficult to construct the activity profile for such a long interval from our data because it is difficult to take into account all observational effects and correctly eliminate them. So Fig. 7 does not represent the real activity profile, but only the uncorrected distribution of selected Taurid fireballs during the whole interval of activity. As described in the following sections, we identified three different groups of Taurids in our data set. These are the regular Southern and Northern Taurids (designated as S TAU and N TAU, respectively) and a new branch of Southern Taurids, which we designate S TAU (SB). As shown later, this new branch was responsible for the enhanced activity. From Fig. 7 we can see that enhanced Taurid activity caused by this new branch of Southern Taurids fireballs started on October 24, culminated around November 5 and terminated on the night of November $16 / 17$. The S TAU (SB) was not observed after this date even though on November 18 and 21-23 were at least partly clear nights with good observing conditions. From Fig. 7 we also see that the enhanced activity increased gradually with several days of very high activity at the turn of October and November. This interval was strongly affected by the full Moon period, so fainter fireballs were below the sensitivity limit of the digital all-sky cameras and radiometers especially in the end of October. Therefore the number of fireballs on these nights may be underestimated. On the other hand, the relatively steep decrease of activity after November 5 seems to be real. With regard to regular $\mathrm{S}$ and $\mathrm{N}$ Taurids, they are quite uniformly spread over the entire interval of observed activity.

\section{The 2015 Taurid data}

The total number of Taurid fireballs recorded photographically by our instruments at least from two stations in 2015 was about 200. This is much more than we recorded in any previous year. 


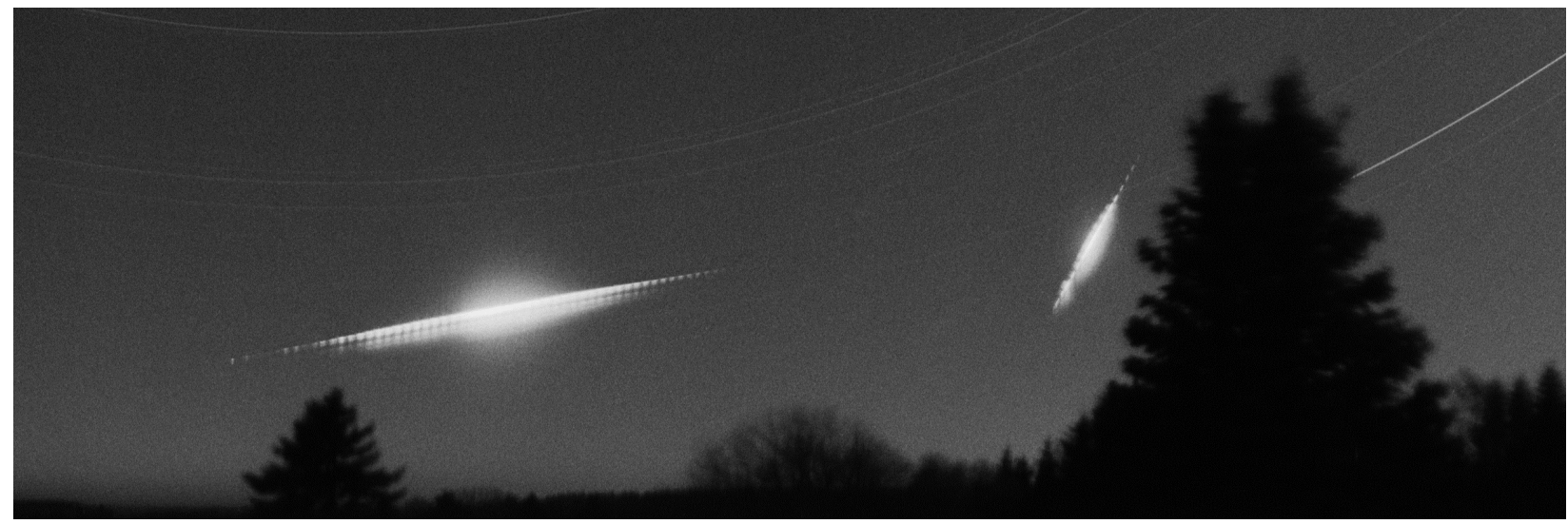

Fig. 8. Detailed view of the two brightest Taurids far over Poland recorded by the AFO (analog camera) at station Polom.

The main reason for this is evidently the unusually high Taurid activity, but it is also caused by much more efficient observational system and also by a quite long period of relatively good weather. For this study we selected 144 Taurids with complete information about heliocentric orbits of individual meteoroids and their physical properties as well. Our data set is unique not because of the total number of used meteors but the high precision of the data for each individual case, which was obtained by with high-resolution cameras and radiometers and elaborated reduction methods. Meteor records were reduced one-by-one and all steps in the measurement and computation process were under careful human supervision.

Before going to the statistical analysis of the whole data set, we describe in more detail some remarkable fireballs.

\subsection{Exceptional cases}

\subsubsection{EN311015_180520 and EN311015_231301 - two brightest Taurids}

It is a well-known fact that Taurids are quite rich in bright fireballs. However, 2015 was also in this aspect exceptional and our cameras recorded several very bright Taurids during the whole period of activity. Altogether 24 Taurids were brighter than -10 absolute magnitude and 10 were similarly bright or even brighter than the full Moon. Moreover, two of those, both observed in the first half of the night of October 31, are really remarkable not only in this data set, but in all Taurids that we have recorded within the EN until now. Both are shown in Fig. 8, where a small part of the all-sky image is shown. This image was taken from station Polom by the AFO, i.e., on sheet film, where one exposure was taken per night. Both bolides were observed in a similar (northern) direction and flew over northern and central Poland, respectively. The brighter bolide, which reached a peak absolute magnitude of -18.6 (on the left), occurred at 18:05:20 UT and the second, with peak magnitude of -15.8 , occurred $5 \mathrm{~h} 7 \mathrm{~min}$ and $41 \mathrm{~s}$ later at 23:13:01 UT. This is the reason why their directions of flight differ, although both bolides had practically the same radiant. The first was so bright that it belonged to the superbolide category. This spectacular Taurid bolide was caused by a meteoroid with initial mass more than $1000 \mathrm{~kg}$, i.e., a metersized object. Because of its enormous brightness, clear skies over large parts of Central Europe, and convenient time of its occurrence (it was an unusually nice Saturday evening), thousands of eyewitnesses were fascinated by this extraordinary natural event. We obtained more reports of one bolide than ever before. Apart from plenty of visual observations, all DAFOs and AFOs in our network (at 15 stations) recorded it, which was crucial for reliable description of this superbolide. In addition to our own photographic and radiometric records, we used also two casual images. The first one is a high-resolution digital image from Studénka, Czech Republic, which was obtained from amateur astronomer B. Pelc. The second digital image was taken by G. Zieleniecki at Czernice Borowe, Poland, and was freely available on the internet. Altogether we used 13 most suitable photographic and 5 radiometric records. The situation with the second, much smaller, meteoroid was similar. It was also recorded by all our cameras at all 15 stations, and we obtained also 4 high-resolution casual digital images from northern region of the Czech Republic. These images, which we also partially used, were taken by T. Chlíbec at Klínovec, L. Sklenár from Kunčice and Labem, D. Ščerba from Dolní Údolí, and L. Shrbený from Říčany; this record also includes a spectrum of the bolide. In this case we used the best 12 photographic and 4 radiometric records for final analysis.

Since these fireballs were exceptional, we modeled them with our semiempirical fragmentation model (Borovička et al. 2013). The model fits radiometric curves and deceleration. This way we obtained more reliable initial masses of meteoroids (1300 kg and $34 \mathrm{~kg}$, respectively) and insight into their atmospheric fragmentation. Both meteoroids were effectively destroyed high in the atmosphere under dynamic pressures $<0.05 \mathrm{MPa}$. In both cases a small fragment $(<1 \mathrm{~kg})$ survived the initial destruction and fragmented further under pressures of $\sim 0.1 \mathrm{MPa}$. In comparison with other bright bolides (Borovička et al. 2017), both Taurids were extremely fragile.

Simultaneously with our network, both bolides were also recorded by the cameras of the Polish Fireball Network. These data were analyzed independently and were published by Olech et al. (2016). Because our data differ from their data, we provide here our complete results and compare them to those reported in Olech et al. (2016). Atmospheric trajectories are given in Table 1, light curves in Fig. 9, and heliocentric orbits in Table 2. When computing the local azimuth and slope of the trajectory, we took into account both the curvature of the Earth and the curvature of the bolide trajectory due to gravity, which was significant for EN311015_180520 (change of direction of flight by $0.17^{\circ}$ over the recorded length). Azimuths are measured from the south clockwise. The apparent radiants given in Table 2 are valid for the average points on the trajectories.

As shown later in this paper and that of Olech et al. (2016) (in fact that paper is based only on these two bolides) data about these big bolides are of great importance. Since it may not be 
Table 1. Atmospheric trajectory data for the EN311015_180520 (left) and EN311015_231301 (right) bolides.

\begin{tabular}{l|ccc|ccc}
\hline \hline & Beginning & $\begin{array}{c}\text { Max. } \\
\text { bright. }\end{array}$ & Terminal & Beginning & $\begin{array}{c}\text { Max. } \\
\text { bright. }\end{array}$ & Terminal \\
\hline Height $(\mathrm{km})$ & $114.724 \pm 0.025$ & 80.8 & $57.644 \pm 0.030$ & $120.026 \pm 0.030$ & 74.4 & $57.305 \pm 0.016$ \\
Velocity $\left(\mathrm{km} \mathrm{s}^{-1}\right)$ & $33.07 \pm 0.03$ & 33.07 & $22 \pm 2$ & $32.56 \pm 0.09$ & 32.53 & $30 \pm 2$ \\
Longitude $\left({ }^{\circ} \mathrm{E}\right)$ & $18.46416 \pm 0.00018$ & 16.927 & $15.82901 \pm 0.00020$ & $18.18064 \pm 0.00026$ & 18.101 & $18.07087 \pm 0.00014$ \\
Latitude $\left({ }^{\circ} \mathrm{N}\right)$ & $53.60723 \pm 0.00050$ & 53.553 & $53.50244 \pm 0.00048$ & $52.13173 \pm 0.00060$ & 52.430 & $52.54390 \pm 0.00033$ \\
Slope $\left(^{\circ}\right)$ & $18.574 \pm 0.014$ & 17.71 & $17.148 \pm 0.034$ & $53.28 \pm 0.04$ & 53.00 & $52.89 \pm 0.10$ \\
Azimuth $\left({ }^{\circ}\right)$ & $267.240 \pm 0.013$ & 266.00 & $265.120 \pm 0.015$ & $350.78 \pm 0.04$ & 350.72 & $350.69 \pm 0.05$ \\
Time ${ }^{1}(\mathrm{~s})$ & -1.07 & 2.22 & 4.68 & -0.07 & 1.68 & 2.36 \\
Total length $(\mathrm{km})$ & & 186.3 & & & 78.5 & \\
\hline
\end{tabular}

Notes. ${ }^{(1)}$ Time zero corresponds to 18:05:18 UT for EN311015_180520 and 23:13:00 UT for EN311015_231301.

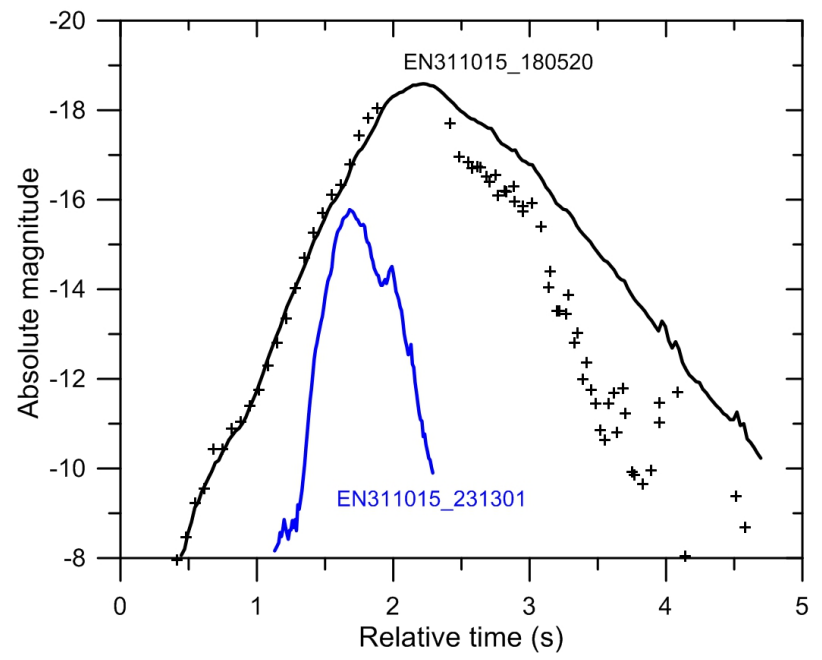

Fig. 9. Calibrated radiometric light curves of bolides EN311015_180520 and EN311015_231301 (solid curves). For EN311015_180520 data from two imaging cameras are also given (crosses). After bolide maximum, most of radiometric signal was produced by a stationary trail. Camera data contain only the bolide moving further down. Time zero corresponds to 18:05:18 UT for EN311015_180520 and 23:13:00 UT for EN311015_231301.

simple to distinguish which data set is correct, we carry out an analysis of the differences. The positions of the recorded beginning and end points of the bolide depend on the sensitivity of the instrument and the observing conditions. Nevertheless, when plotting the trajectory of the first bolide on the map, the solution of Olech et al. (2016) is shifted about $1.8 \mathrm{~km}$ to the north. As for the observed apparent radiant, there is a difference of $0.65^{\circ}$ in declination, i.e., 10 times their quoted uncertainty $(\sigma)$. For the second bolide, the larger difference is in right ascension $\left(0.24^{\circ}, 4 \sigma\right)$ and especially in entry velocity, which is larger by $0.6 \mathrm{~km} \mathrm{~s}^{-1}(6 \sigma)$ in Olech et al. (2016). Although our data were obtained from large distances, our results are based on large number of records (in both cases more than 10) and the solutions for both bolides are very consistent. The photograph from Czernice Borowe, when combined with our cameras, provides convergence angles in excess of $60^{\circ}$ for the first bolide. Czech cameras have mutual convergence angles up to $15^{\circ}$. For the second bolide the situation is even better, although the velocity was more difficult to measure. So there is no reason, why our
Table 2. Apparent and geocentric radiants and orbital elements (J2000.0) for the EN311015_180520 (left) and EN311015_231301 (right) meteoroids.

\begin{tabular}{c|c|c}
\hline & EN311015_180520 & EN311015_231301 \\
\hline Time $(\mathrm{UT})$ & $18^{\mathrm{h}} 05^{\mathrm{m}} 20.0^{\mathrm{s}} \pm 0.1^{\mathrm{s}}$ & $23^{\mathrm{h}} 13^{\mathrm{m}} 01.5^{\mathrm{s}} \pm 0.1^{\mathrm{s}}$ \\
$\alpha_{R}\left(^{\circ}\right)$ & $50.126 \pm 0.009$ & $51.853 \pm 0.022$ \\
$\delta_{R}\left(^{\circ}\right)$ & $16.452 \pm 0.016$ & $15.66 \pm 0.04$ \\
$\mathrm{v}_{\infty}\left(\mathrm{km} \mathrm{s}^{-1}\right)$ & $33.068 \pm 0.030$ & $32.56 \pm 0.09$ \\
$\alpha_{G}\left({ }^{\circ}\right)$ & $51.692 \pm 0.010$ & $51.445 \pm 0.022$ \\
$\delta_{G}\left({ }^{\circ}\right)$ & $14.592 \pm 0.017$ & $14.49 \pm 0.04$ \\
$\mathrm{v}_{G}\left(\mathrm{~km} \mathrm{~s}^{-1}\right)$ & $30.869 \pm 0.032$ & $30.59 \pm 0.10$ \\
$\mathrm{v}_{H}\left(\mathrm{~km} \mathrm{~s}^{-1}\right)$ & $37.32 \pm 0.02$ & $37.34 \pm 0.06$ \\
$a(\mathrm{AU})$ & $2.250 \pm 0.009$ & $2.258 \pm 0.027$ \\
$e$ & $0.8724 \pm 0.0006$ & $0.8689 \pm 0.0020$ \\
$q(\mathrm{AU})$ & $0.28715 \pm 0.00032$ & $0.2960 \pm 0.0010$ \\
$Q(\mathrm{AU})$ & $4.212 \pm 0.018$ & $4.22 \pm 0.05$ \\
$\omega\left(^{\circ}\right)$ & $121.687 \pm 0.022$ & $120.62 \pm 0.06$ \\
$\Omega\left(^{\circ}\right)$ & 37.791 & 38.005 \\
$i\left(^{\circ}\right)$ & $5.707 \pm 0.023$ & $5.62 \pm 0.06$ \\
$P(\mathrm{yr})$ & $3.375 \pm 0.020$ & $3.39 \pm 0.06$ \\
Perihelion & $2012-07-26 \pm 7 \mathrm{~d}$ & $2012-07-20 \pm 22 \mathrm{~d}$ \\
$\mathrm{TP}_{\mathrm{Jup}}$ & $2.952 \pm 0.009$ & $2.953 \pm 0.028$ \\
\hline
\end{tabular}

Notes. Time is given for the average point of the recorded trajectory.

results should be different by more than the standard deviations given in Table 1.

There is also at least a $3-5 \mathrm{~s}$ difference in the reported time of appearance of the first fireball. Our radiometers are continuously corrected by PPS pulse of GPS and their timing precision is in millisecond range. A nice example how our radiometers are synchronized is shown in Fig. 4. Another discrepancy is in the determination of the maximum absolute brightness for both bolides. While Olech et al. (2016) determined the maximum absolute brightness $-16.0 \pm 0.4$ mag for the first bolide, we found that it reached $-18.6 \pm 0.2 \mathrm{mag}$. Our method described in Sect. 3 relies on the linearity of radiometers even for strong signals. Results from five independent radiometers were in perfect 


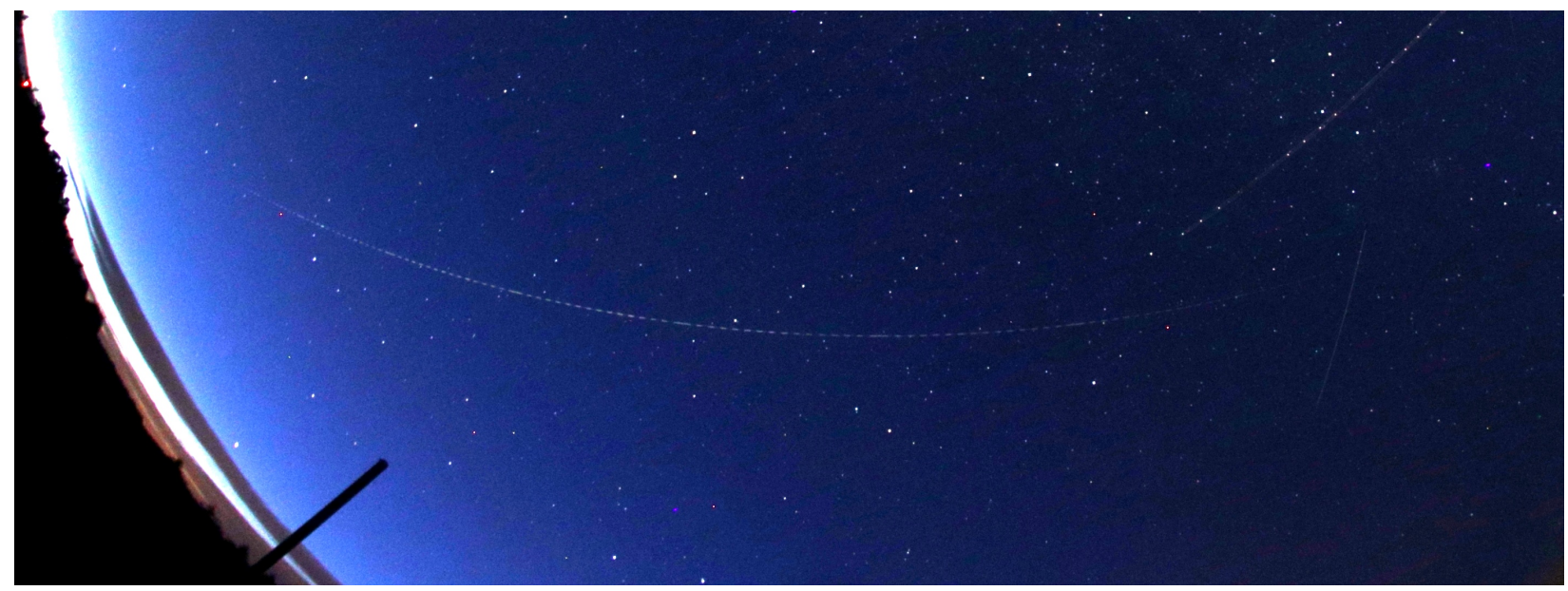

Fig. 10. Detailed view on the very long Taurid fireball recorded by the DAFO (digital camera) at station Kunžak.

agreement. Similarly the brightness of the second bolide was underestimated by Olech et al. (2016) by about $1 \mathrm{mag}$.

Regardless of these differences in the directly determined trajectory parameters, we found another discrepancy in the calculation of orbital elements in Tables 3-5 of Olech et al. (2016). We obtained significantly different results than those published in Tables 5 and 6 of Olech et al. (2016) when we took their input values of initial velocity, apparent radiant position (which we recalculated from $\mathrm{J} 2000.0$ to the date that the bolides occurred) time, and mean position for both bolides from their Tables 3 and 4 and used our program for orbital calculation; orbits from this program were independently validated for example in Clark \& Wiegert (2011). We found the following differences for the EN311015_180520 bolide (computed minus published): $\Delta a=0.0077 \mathrm{AU}, \Delta e=0.0057, \Delta \omega=1.3^{\circ}(!), \Delta i=0.07^{\circ}$, and $\Delta P=0.019$ yr. For the EN311015_231301 bolide, differences are $\Delta a=0.1287 \mathrm{AU}(!), \Delta e=0.0087, \Delta \omega=0.1^{\circ}, \Delta i=0.035^{\circ}$, and $\Delta P=0.20 \mathrm{yr}$. Some of these differences are really high, namely $1.3^{\circ}$, in argument of perihelion for the first bolide and especially $0.128 \mathrm{AU}$ in semimajor axis for the second bolide. With the radiant and velocity given by Olech et al. (2016) this bolide would be far from the 7:2 resonance with Jupiter, nevertheless, their published orbit puts it in the resonance.

\subsubsection{EN061115_164758: an almost horizontal Taurid}

On November 6, 2015 during dawn, just after the Taurid radiant rose above the horizon, a relatively faint Taurid fireball of -5.1 maximum absolute magnitude traveled over a large part of sky and was observed by several stations in the SW part of our network. The sky was not completely dark, especially from the stations in western part of the network, which were closest to the fireball trajectory. However, thanks to the higher sensitivity of the digital cameras, this extremely long fireball was nicely recorded on three stations, Kunžak, Martinsberg and Kuchařovice, which enabled us to describe this exceptional Taurid accurately. Owing to its small slope which was $7.7^{\circ}$ at the beginning and during the flight decreased to only $5.6^{\circ}$, the recorded fireball trajectory was extremely long, i.e., exactly $258.7 \mathrm{~km}$, and its flight lasted $8.5 \mathrm{~s}$. It is the longest Taurid fireball we have ever recorded, both in duration and length. Thanks to a large amount of data points, this Taurid has the best dynamic data and the trajectory, i.e., also radiant, is also very precise. The initial velocity of $31.285 \mathrm{~km} \mathrm{~s}^{-1}$ was determined with a precision of $\pm 7 \mathrm{~m} \mathrm{~s}^{-1}$. As for the brightest Taurid described in Sect. 4.1.1, we took into account the curvature of the trajectory of the bolide due to gravity, which was significant for such a long and low inclined fireball (change of direction of flight by $0.18^{\circ}$ over the recorded length). A detailed view of its luminous flight taken by the DAFO at Kunžak station is shown in Fig. 10. Additional information about this fireball is given in Tables A.1 and A.2.

\subsection{Radiants and orbits}

In this section the radiants, velocities, and heliocentric orbits of all 144 fireballs are evaluated. All elements in this paper are given for equinox J2000.0. The data are presented in Table A.1. Figure 11 shows the dependency of geocentric radiant and velocity on solar longitude (i.e., the longitude of the Sun at the time of fireball observation). Thirteen fireballs were classified as Northern Taurids. They can be easily recognized by their radiant lying to the north of the ecliptic. All other fireballs belong to Southern Taurids. Among them, a well-defined structure can be recognized, where the radiant position and velocity are strict linear functions of solar longitude. We call this structure a new branch. Evidently, this branch was responsible for the enhanced Taurid activity in 2015.

Regular Taurids also exhibit radiant motion but the spread of individual radiants is much larger than for the new branch. For the new branch, we found the following relationships:

$\alpha_{\mathrm{g}}=46.99^{\circ}+0.554 \cdot\left(\lambda_{\odot}-210^{\circ}\right)$
$\delta_{\mathrm{g}}=14.00^{\circ}+0.060 \cdot\left(\lambda_{\odot}-210^{\circ}\right)$
$v_{\mathrm{g}}=32.90-0.293 \cdot\left(\lambda_{\odot}-210^{\circ}\right)$,

where $\alpha_{\mathrm{g}}$ and $\delta_{\mathrm{g}}$ are the right ascension and declination, respectively, of the geocentric radiant (J2000.0), $v_{\mathrm{g}}$ is the geocentric velocity in $\mathrm{km} \mathrm{s}^{-1}$, and $\lambda_{\odot}$ is solar longitude (J2000.0). Although we defined the new branch on the basis of orbital elements rather than radiants and velocities (see below), all fireballs of the new branch had the radiant right ascension within $1.3^{\circ}$ and declination within $0.7^{\circ}$ from (1) and (2). The velocities were within $0.9 \mathrm{~km} \mathrm{~s}^{-1}$ from relationship (3). We point out that this spread is real. The precision of most of our data, as demonstrated in Sect. 3 , is $<0.05^{\circ}$ in the radiant position and $0.1 \mathrm{~km} \mathrm{~s}^{-1}$ in the velocity.

Fireballs from the branch were observed between solar longitudes $211^{\circ}-234^{\circ}$ (October 25-November 17). Taurids 

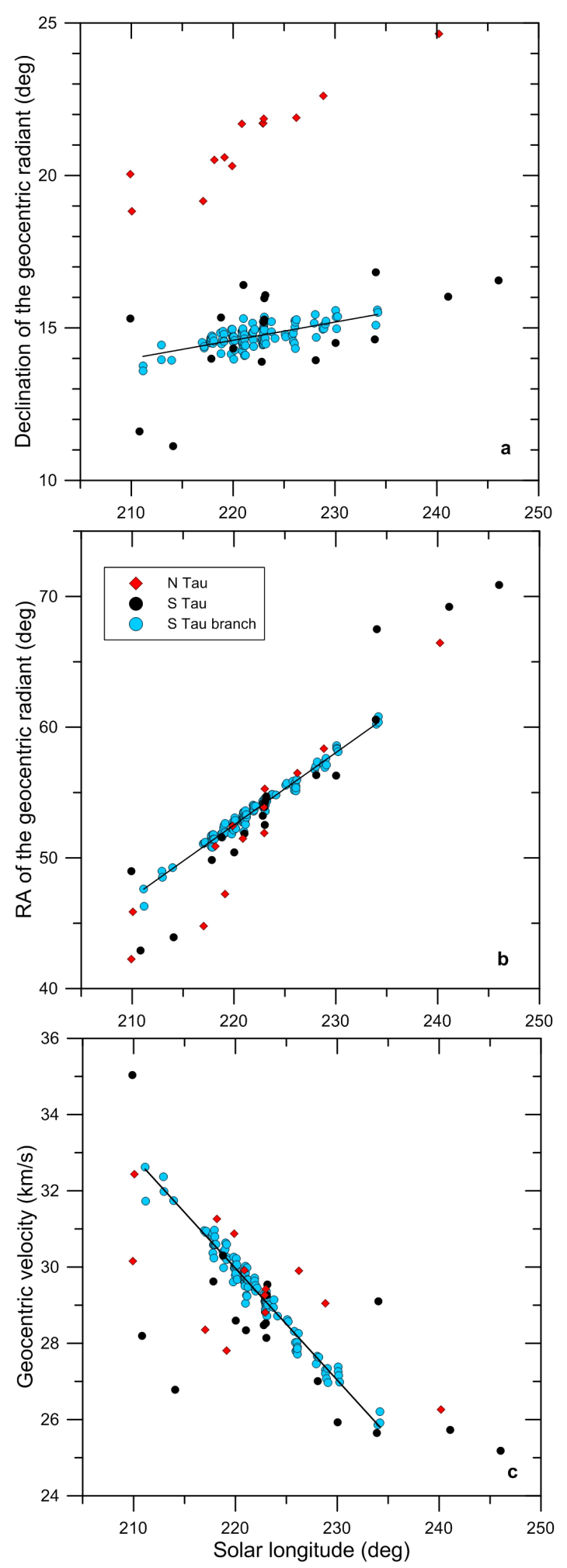

Fig. 11. Position of geocentric radiant and geocentric velocity as a function of solar longitude for Taurids observed in 2015. Northern and Southern Taurids are shown by different symbols and the Southern Taurids belonging to the new branch are highlighted in light blue. The error of the data is smaller than the size of the symbols in most cases.

observed before and after these dates belong to the background population of Northern and Southern Taurid streams.

Longitude of perihelion, inclination, eccentricity, and perihelion distance as a function of solar longitude are plotted in
Fig. 12. Most notably, there is a concentration of orbits with longitude of perihelion, $\pi$ ( $\pi=\Omega+\omega$, where $\Omega$ is longitude of ascending node and $\omega$ is argument of perihelion), at $158^{\circ} \pm 2^{\circ}$ (Fig. 12a). There is only a weak correlation with solar longitude. Similarly, there is a concentration of orbits with inclinations of $5.5^{\circ} \pm 1^{\circ}$ (Fig. 12b). Regular Taurids show much larger spread, $145-175^{\circ}$ in $\pi$ and $2-7^{\circ}$ in inclination.

Eccentricities and perihelion distances of the members of the new branch are steep functions of solar longitude (Figs. 12c, d), i.e.,

$e=0.901-0.00403 \cdot\left(\lambda_{\odot}-210^{\circ}\right)$

$q=0.224+0.0092 \cdot\left(\lambda_{\odot}-210^{\circ}\right)$.

All eccentricities lie within 0.012 from (4) and perihelia lie within $0.027 \mathrm{AU}$ from (5). Again, regular Taurids show much larger scatter.

The new branch is best recognized in the plot of longitude of perihelion, $\pi$, versus latitude of perihelion, $\beta(\sin \beta=\sin \omega \sin i$, where $i$ is inclination), presented in Fig. 13. We can state that the new branch has $\pi$ between $155.9-160^{\circ}$ and $\beta$ between $4.2-$ $5.7^{\circ}$. For regular Southern Taurids the observed spread in $\beta$ is 2.5-6.5 . Northern Taurids have negative $\beta$.

Semimajor axes are plotted in Fig. 14. For regular Taurids, they lie between 1.9 and 2.4 AU. According to the model of Asher \& Clube (1993), the enhanced activity is caused by meteoroids trapped in the 7:2 resonance with Jupiter. The resonance is located at 2.256 $\mathrm{AU}$ and extends from about 2.231 $\mathrm{AU}$ to 2.281 AU (Asher \& Clube 1993). With two exceptions, the semimajor axes of all meteoroids with longitudes and latitudes of perihelia within the above-defined limits fall in the 7:2 resonance. Only two meteoroids had significantly lower semimajor axes, 2.15-2.16 AU. We consider them to be interlopers from the background population of Southern Taurids, although the 15:4 resonance located at 2.155 $\mathrm{AU}$ might be at work here.

On the contrary, some Southern Taurids with perihelia outside the new branch limits were also in the 7:2 resonance. As seen in Fig. 13, all of the Souther Taurids had an orientation of perihelia relatively close to the new branch. Nevertheless, some Northern Taurids were in the 7:2 resonance as well and they were far from the new branch.

There is no correlation between semimajor axis and solar longitude. The Tisserand parameter with respect to Jupiter increases with solar longitude from 2.9 to 3.1 within the new branch. This is due to the decreasing eccentricity. The often cited boundary at $T_{\text {Jup }}=3$ or 3.05 (e.g., Tancredi 2014) has no significance in this case.

According to the above definitions based on perihelion orientation and semimajor axis, there are 13 Northern Taurids in our data set, 18 regular Southern Taurids, and 113 members of the new branch.

It is evident that the new branch represents an orbital structure that is much more compact than regular Taurids. Since the activity of the new branch lasted almost one month, it cannot, however, be a narrow filament. In order to visualize the new branch, we plotted selected orbits covering the whole activity period in Fig. 15. Unlike usual meteoroid streams, where the orbits near perihelion largely overlap, here we see a concentric ring of orbits near perihelion, which is more than $0.2 \mathrm{AU}$ wide. As the Earth moves around the Sun, it encounters first the orbits with smaller perihelia and larger eccentricities. With increasing solar longitude, orbits with progressively larger perihelia and smaller eccentricities are encountered. Since all of the semimajor axes are similar, eccentric orbits have larger aphelia than less eccentric orbits and the orbits therefore intersect at about 3.6 AU. 

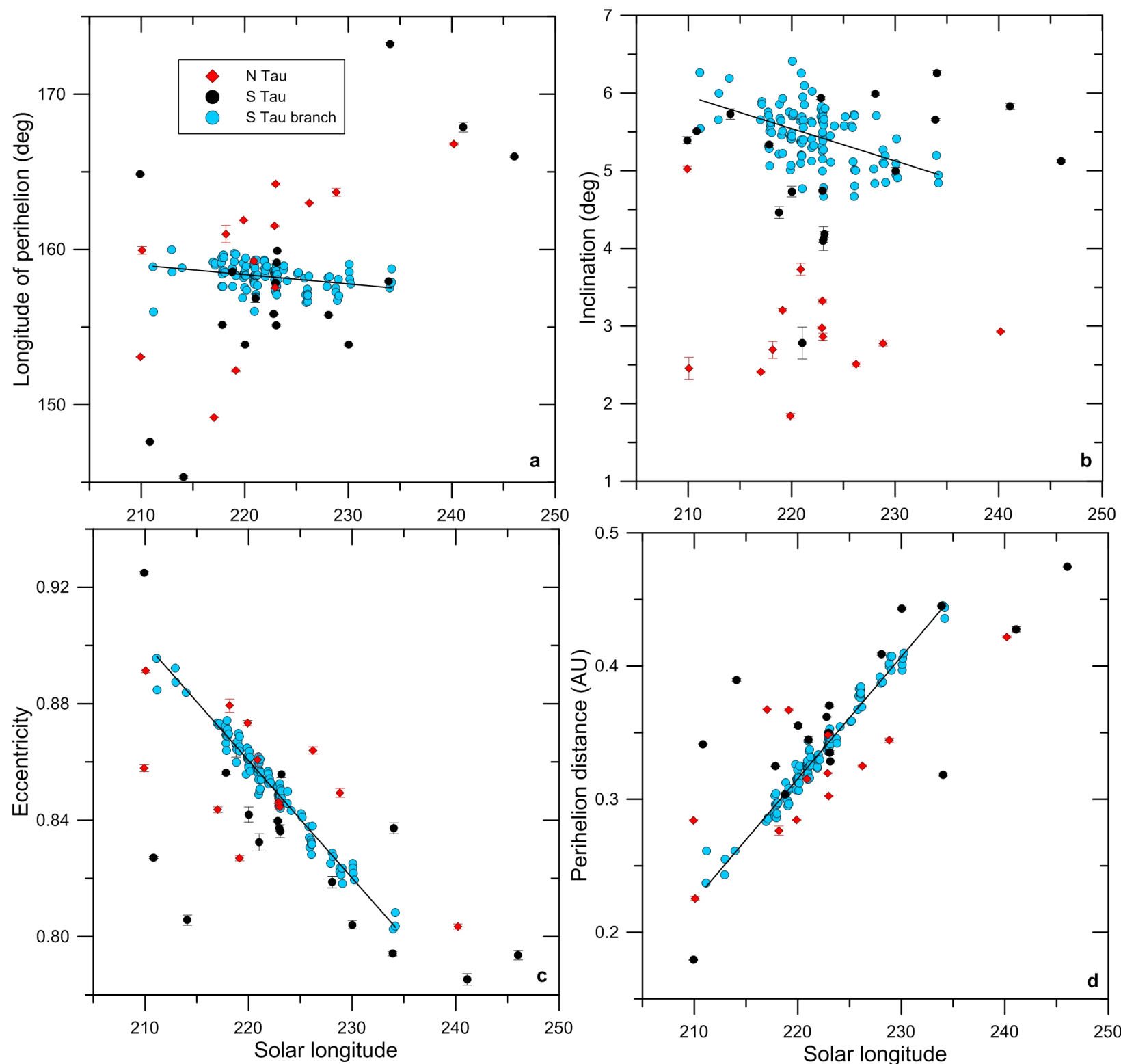

Fig. 12. Selected orbital elements as a function of solar longitude for Taurids observed in 2015. The symbols are the same as in Fig. 11. Errors are in most cases smaller than symbol sizes and for clarity are plotted only for regular Taurids.

\subsection{Physical properties}

The Taurids in our sample reached maximum absolute magnitude between -2 and -18.6 . The photometric masses range from $0.1 \mathrm{~g}$ to $1300 \mathrm{~kg}$, i.e., there is a range of 7 orders of magnitude in mass. The mass distribution is given in Fig. 16, which shows that the new branch has a higher proportion of massive meteoroids. The data in Fig. 16 are biased because brighter meteors could be observed over large distances and under worse conditions than faint meteors, nevertheless, the bias is the same for all branches.

The beginning, maximum brightness, and end heights of all studied fireballs are plotted as a function of photometric mass in Fig. 17. These heights are good proxies to meteoroid structure, although they depend to some extent on observational circumstances (e.g., range to the fireball) and on the slope of the trajectory. Beginning heights show no dependence on mass and are generally between 90 and $110 \mathrm{~km}$. For consistency we use only data from digital all-sky cameras in the plot. The two brightest fireballs were captured by the narrow-field cameras at higher altitudes (see Table 1). On the other hand, both these fireballs were located far from the all-sky cameras; the beginning of EN 311015_180520 was $390 \mathrm{~km}$ from the closest camera and the beginning of EN 311015_231301 was $270 \mathrm{~km}$ distant. If observed from closer distances, the beginnings would lie somewhat higher.

The maximum and end heights show large scatter. Many fireballs exhibited multiple flares of similar brightness. Nevertheless, there were differences in physical properties of the meteoroids. This fact is mostly evident from the end heights. There are differences of $25 \mathrm{~km}$ or more for meteoroids of similar masses. The expected trend of deeper penetration for larger bodies is only weakly present. The lowest end heights (below $50 \mathrm{~km}$ ) were achieved by two quite small meteoroids. There are no obvious differences in physical properties between different branches of the stream.

Since the end height depends not only on the meteoroid properties but also on trajectory slope and entry speed, the PE 


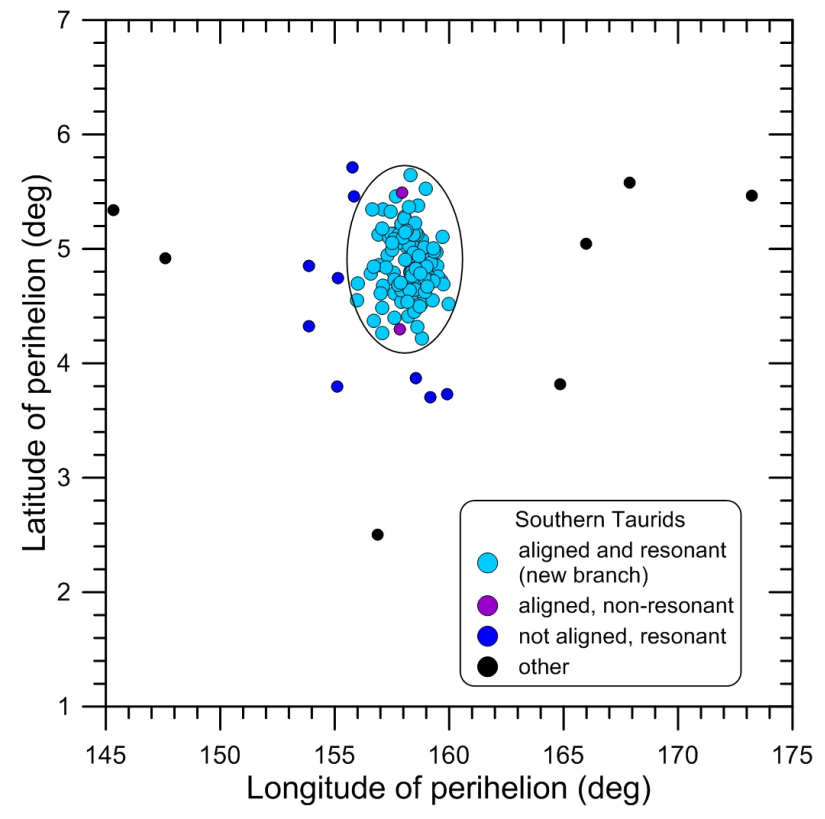

Fig. 13. Orientation of perihelia (latitude versus longitude) for Southern Taurids observed in 2015. This plot was used to define the limits of the new branch, as indicated by the ellipse. The fireballs that fell within these limits but had different semimajor axes (outside the 7:2 resonance) are plotted in purple. The fireballs outside these limits but within the resonance are plotted in dark blue.

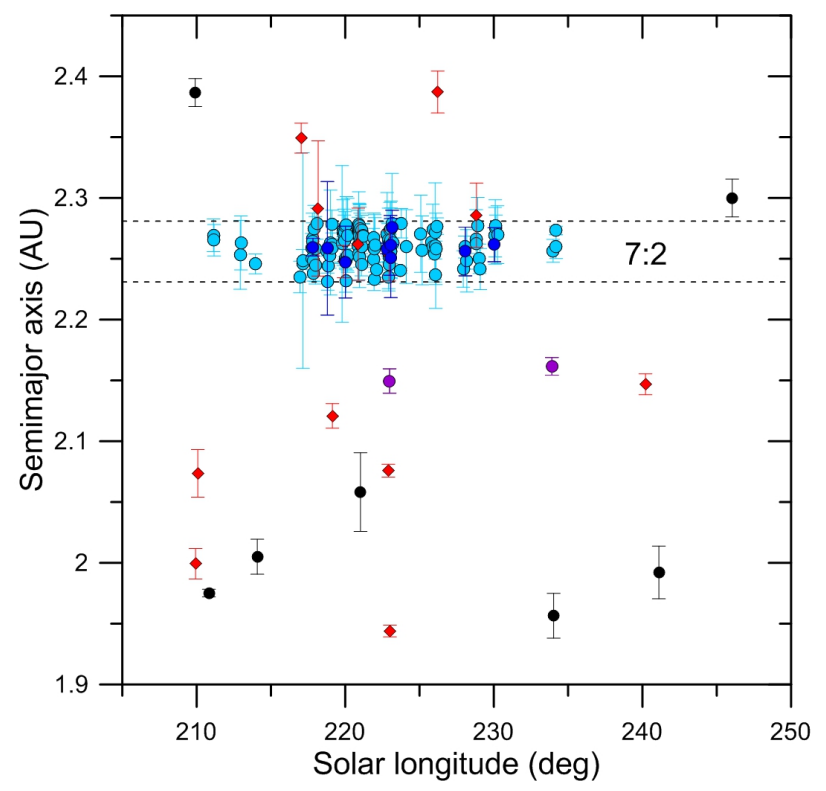

Fig. 14. Semimajor axis as a function of solar longitude for Taurids observed in 2015. For symbol explanation see Figs. 11 and 13. Error bars are plotted for all fireballs. The extent of the 7:2 resonance with Jupiter according to Asher \& Clube (1993) is indicated.

criterion (Ceplecha \& McCrosky 1976), which compensates for these effects, can be used to better evaluate meteoroid strengths. According to the PE criterion, meteoroids are classified into four types: I, II, IIIA, and IIIB (Ceplecha 1988). Type I corresponds to stony meteorites and type IIIB to soft cometary material. Figure 18 shows Taurid PE classification as a function of mass. We can see that Taurids cover all four types, with a clear trend of larger meteoroids being more fragile. Most of meteoroids smaller than $30 \mathrm{~g}$ belong to type II. Some meteoroids

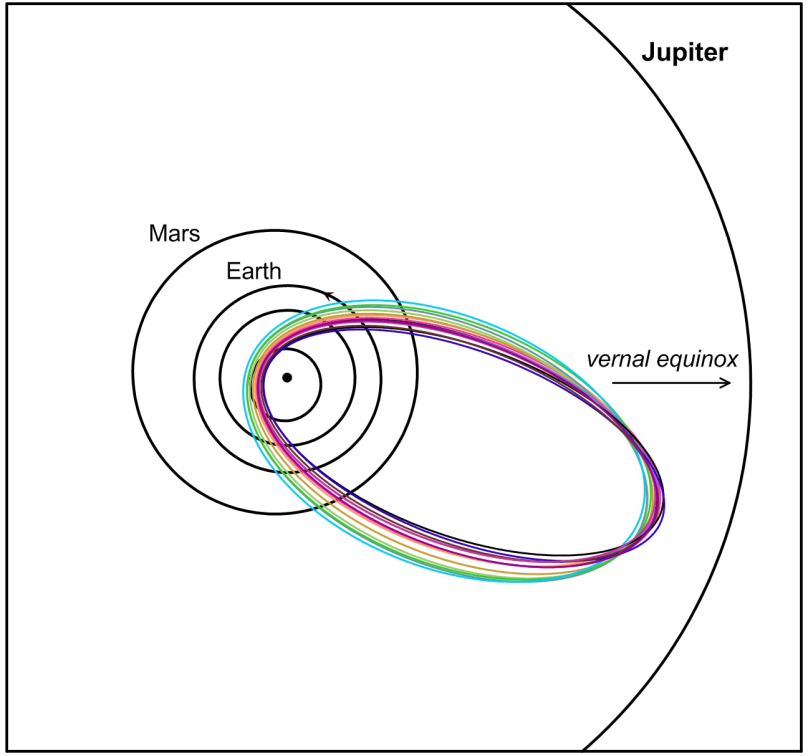

Fig. 15. Selected orbits of the Taurids from the new branch projected to the plane of ecliptic.

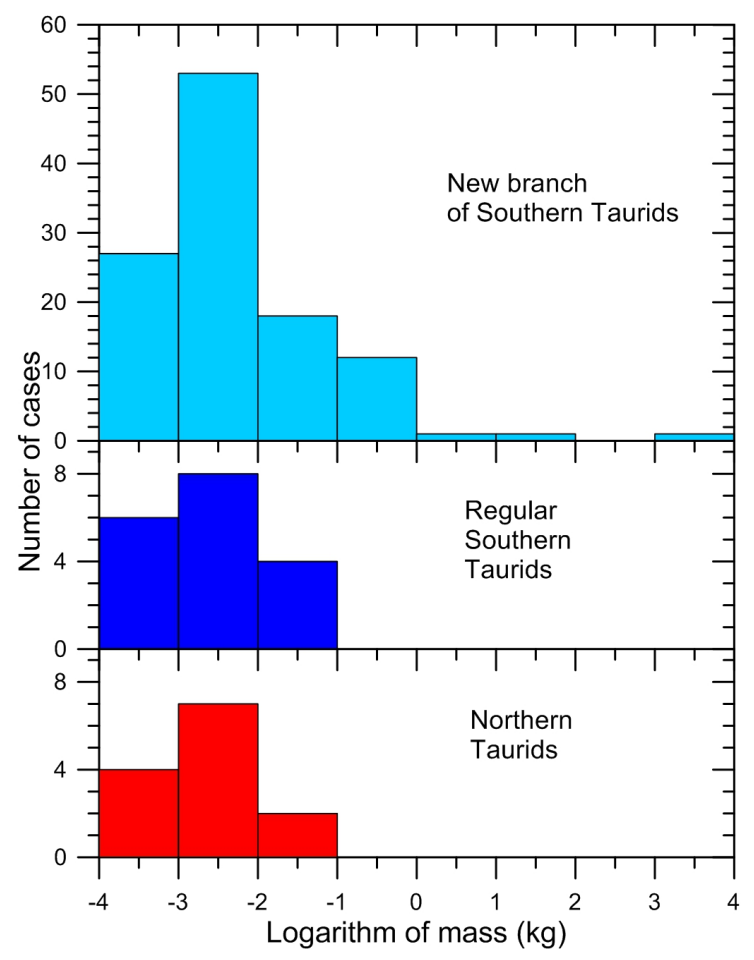

Fig. 16. Number of fireballs as a function of photometric mass for Northern Taurids, regular Southern Taurids, and the new branch.

with masses on the order of one gram clearly belong to type I. On the other hand, most meteoroids above $30 \mathrm{~g}$ belong to type IIIA or IIIB and only type IIIB is present above $300 \mathrm{~g}$. The fact that the two largest meteoroids were very fragile was confirmed by fragmentation modeling (Sect. 4.1.1). Significant differences between small and large meteoroids suggest the existence of some hierarchical structure and will be subject of future studies.

Similar heterogeneity of Taurid physical properties was observed recently by Matlovič et al. (2017). Brown et al. (2013) reported a Taurid that penetrated down to $35 \mathrm{~km}$ and Madiedo et al. (2014) and another one reaching $42.5 \mathrm{~km}$. These authors 


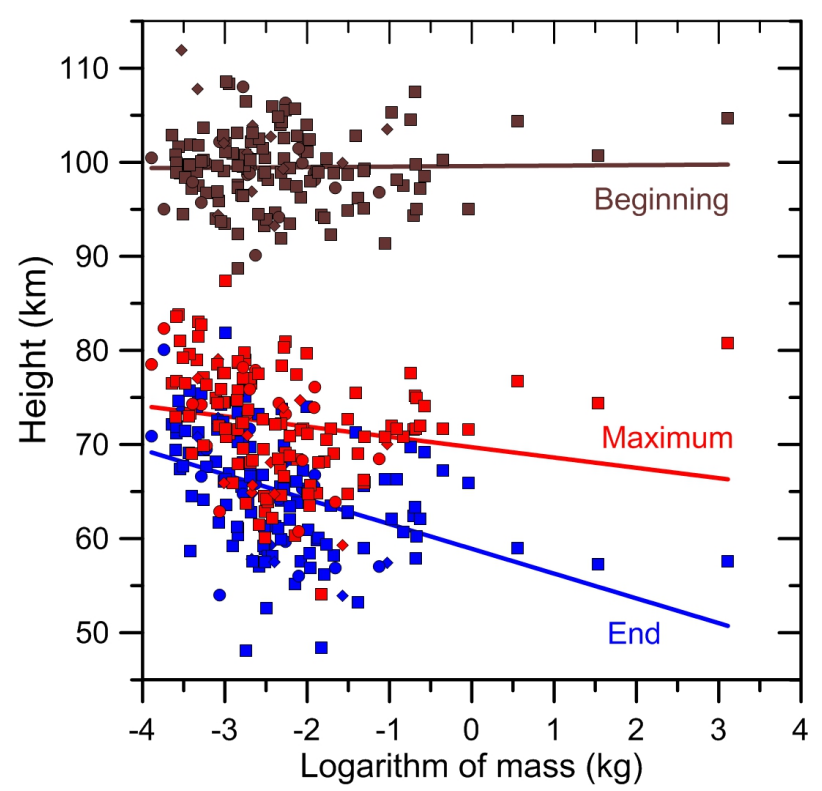

Fig. 17. Fireball heights at beginning, end, and maximum light as a function of photometric mass. Northern Taurids are plotted as diamonds, regular Southern Taurids as circles, and new branch members as squares.

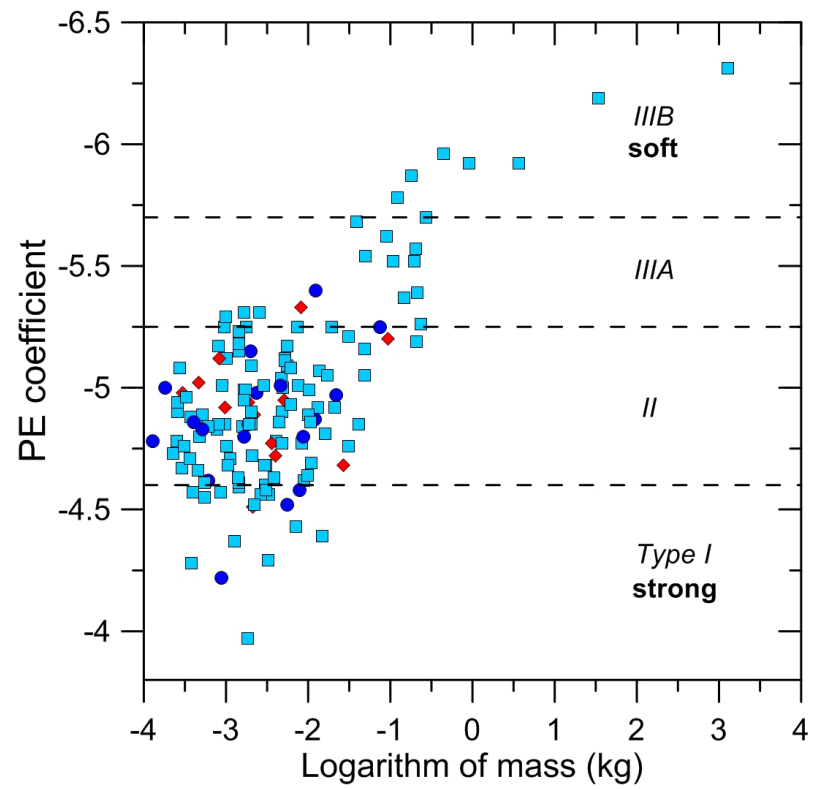

Fig. 18. Value of PE criterion (Ceplecha \& McCrosky 1976) as a function of photometric mass for all observed Taurids. Northern Taurids are plotted as diamonds, regular Souther Taurids as circles, and new branch members as squares. The dashed horizontal lines define the types I, II, IIIA, and IIIB.

suggested that Taurids might drop meteorites. Our data do not seem to support this possibility, since at least a $\sim 1 \mathrm{~kg}$ type I Taurid meteoroid would be needed to produce any meteorites. The SPMN 051010 fireball observed by Madiedo et al. (2014) on October 5, 2010 had a semimajor axis 3.0 AU and perihelion 0.47 AU. It may not be Taurid at all. The SOMN 101031 fireball observed by Brown et al. (2013), with a semimajor axis 2.9 AU, was also not a typical Taurid.

All atmospheric and physical data are given in Table A.2.

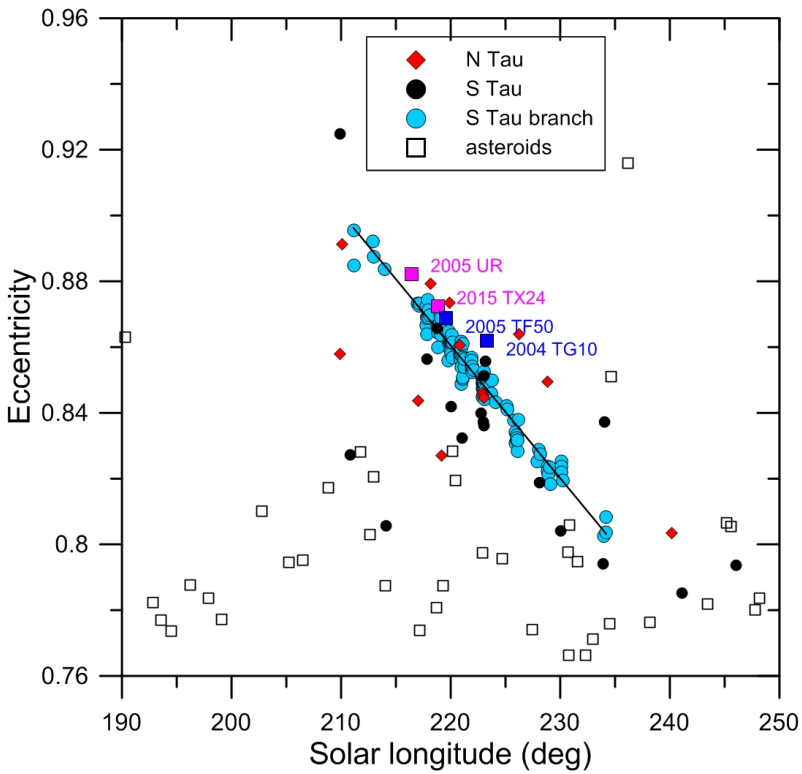

Fig. 19. Orbital eccentricity as a function for solar longitude at the closest approach to the Earth's orbit for 2015 Taurid fireballs and asteroids from JPL database. Asteroids, which are likely related to the new Taurid branch are highlighted in magenta. Asteroids for which the relation to the new branch was considered but not confirmed are shown as filled rectangles. They may be related to other parts of the Taurid complex.

\section{Related asteroids}

We performed a search for asteroids with orbits similar to the new Taurid branch responsible for the enhanced activity in 2015 . For that purpose, asteroids with $q<0.6 \mathrm{AU}, 1.8 \mathrm{AU}<a<$ $2.8 \mathrm{AU}$, and $i<12^{\circ}$ were selected from the JPL Small-Body Database $^{2}$. There are 329 such asteroids known. We then plotted selected orbital elements as a function of solar longitude at Earth Minimum Orbit Intersection Distance (MOID) to be compared with the observed fireballs. For fireballs we used solar longitude at the time of impact as the independent variable. Since the asteroids did not impact Earth and their orbits do not intersect Earth's orbit, we used for comparison the solar longitude, as seen from the asteroid at the time when the asteroid is closest to the Earth's orbit.

Figure 19 shows the comparison plot for eccentricity. We see that there is nearly random distribution of asteroids with eccentricities smaller than 0.84 in the solar longitudes of interest. At higher eccentricities (0.86-0.88), however, there is a noticeable concentration of four asteroids (2005 UR, 2015 TX24, 2005 TF50, and 2004 TG10) near solar longitude of $220^{\circ}$. This concentration overlaps with the new Taurid branch. Moreover, it follows the same trend of decreasing eccentricity with increasing solar longitude.

Other orbital elements are compared in Fig. 20. Perihelion distance is basically a mirror image of eccentricity. Semimajor axes of all four asteroids of interest fall within the Taurid branch range, i.e., also within the 7:2 resonance. As for inclination, only 2015 TX24 falls exactly within the Taurid branch range. The 2005 UR asteroid is somewhat off but only about a half degree from the edge of the Taurid branch. However, Taurid fireballs represent the part of the stream, which intersects Earth's orbit. The whole stream is probably somewhat wider, so we consider

2 http://ssd.jpl.nasa.gov/sbdb_query.cgi, accessed January 25, 2017. 

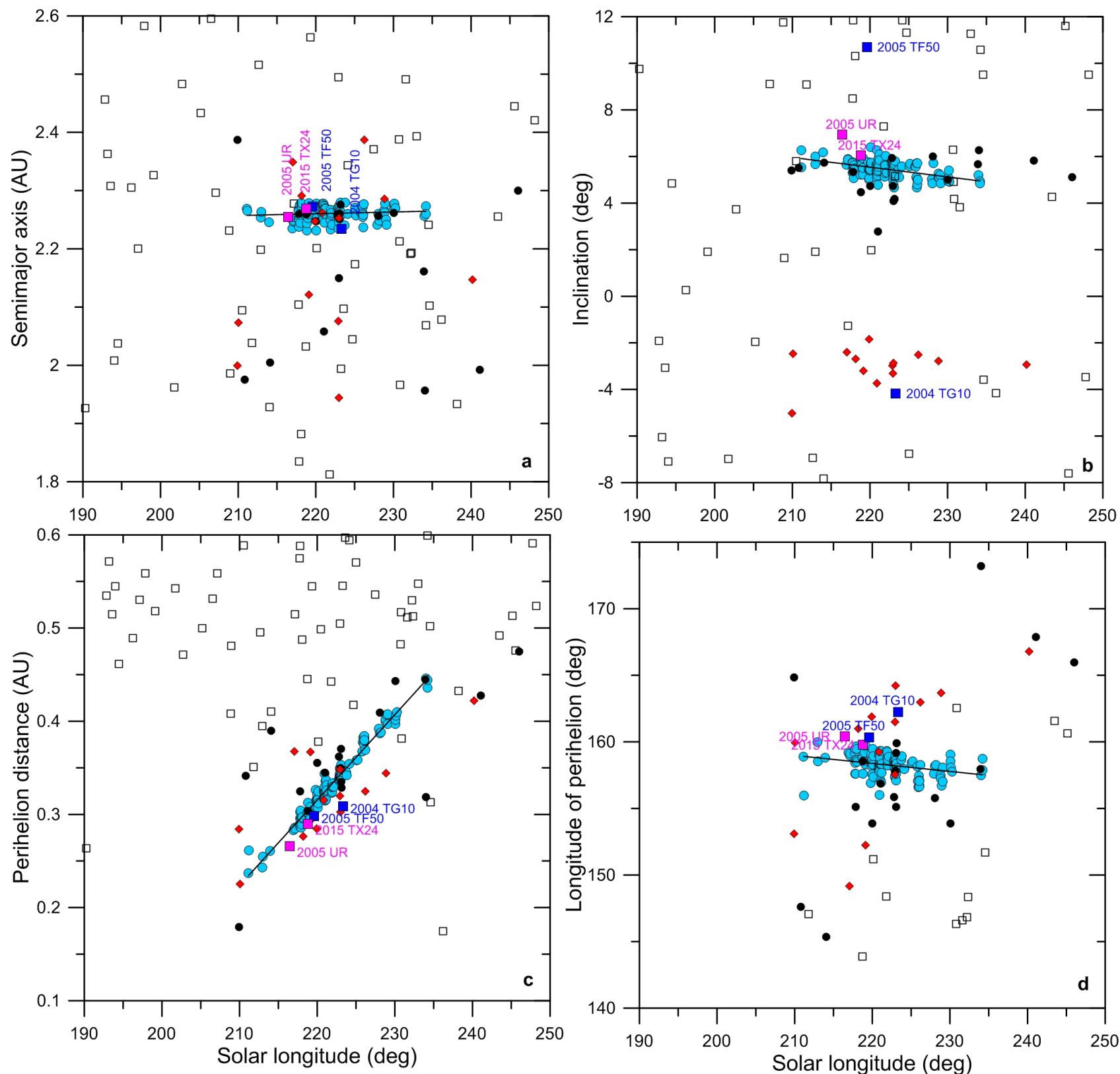

Fig. 20. Semimajor axis, inclination, perihelion distance, and longitude of perihelion as a function for solar longitude at the closest approach to the Earth's orbit for 2015 Taurid fireballs and asteroids from JPL database. Asteroids, which are likely related to the new Taurid branch are highlighted. The symbols are the same as in Fig. 19. The inclinations of Northern Taurids and asteroids with $\Omega>180^{\circ}$, which encounter the Earth near their descending node in October/November, are plotted as negative.

it likely that 2005 UR is also part of the stream. The 2005 TF50 asteroid matches all other elements very well but has an inclination of $10.7^{\circ}$, i.e., more than 4 degrees from the edge of the Taurid branch. On the other hand, the orientation of perihelion is not so far from the new Taurid branch (Fig. 22). But the orbit of 2004 TG10 is oriented in the opposite way relative to the ecliptic. This asteroid may be in fact related to Northern Taurids. At least two asteroids, 2015 TX24 and 2005 UR, are therefore good candidates for direct membership in the new branch of Southern Taurids.

Asteroid 2015 TX24 was discovered by Pan-STARRS 1 on October 8, 2015 and was observed for 18 days in October 2015. It passed closest to the Earth's orbit on October 28, 2015, i.e., during the enhanced Taurid activity. The MOID of the Earth is
0.010 AU. The asteroid has an absolute magnitude of $H=21.5$, which corresponds to diameter 200-300 m, assuming albedo in the range $0.10-0.05$.

Asteroid 2005 UR was discovered by the Catalina Sky Survey on October 23, 2005 and was observed for six days in October 2005. The MOID of the Earth is 0.034 AU. The absolute magnitude is $H=21.6$, i.e., very similar to that of 2015 TX24. Asteroid 2005 UR approached the Earth's orbit at the end of December 2015 and was therefore only $17^{\circ}$ in mean anomaly behind the Taurids observed in 2015. Moreover, as noted by Olech et al. (2016), Taurid activity was also enhanced when the asteroid passed close to the Earth in October 2005.

The orbits of both 2005 UR and 2015 TX24 are plotted in Fig. 21 together with the fireball orbits. There is a good overlap. 


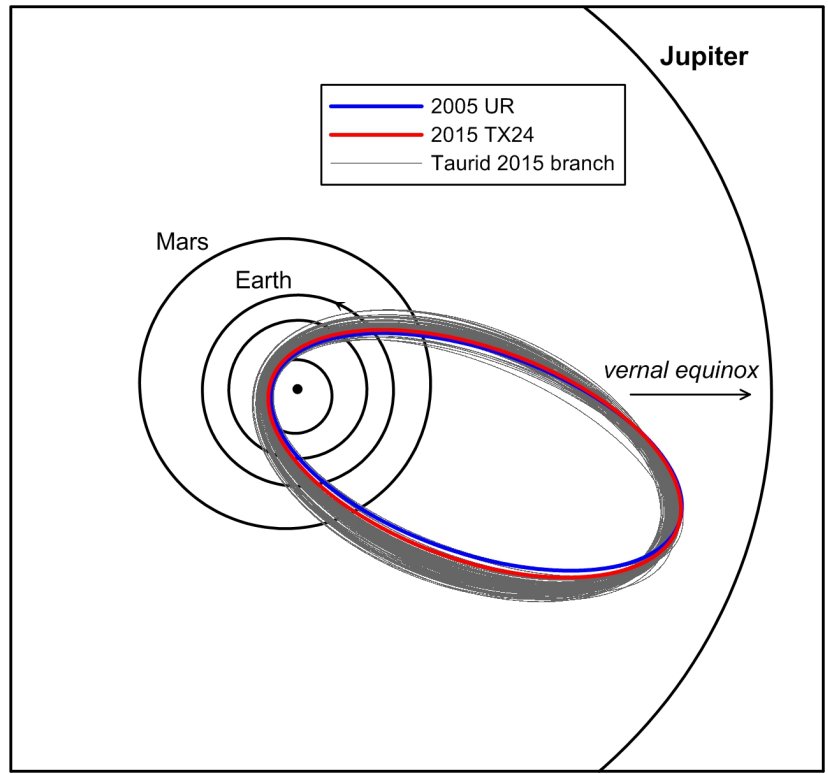

Fig. 21. Orbits of 2005 UR and 2015 TX24 in comparison with all Taurids orbits from the new branch (gray).

Orbital elements of all four asteroids discussed here are given in Table 3. Asteroid $2004 \mathrm{TG} 10$ is a large object with $H=19.4$. The albedo is very low and the diameter was estimated to be $1.40 \pm 0.51 \mathrm{~km}$ (Nugent et al. 2015). The possible relation of this asteroid to the Taurids was suggested already by Jenniskens (2006), Porubčan et al. (2006), and Babadzhanov et al. (2008). Asteroid 2005 TF50, with $H=20.3$, is of intermediate size. Its relation to comet $2 \mathrm{P} /$ Encke and the Taurids was proposed by Porubčan et al. (2006) and Olech et al. (2016).

\section{Discussion}

We presented probably the most precise Taurid orbits obtained to date. Thanks to the sufficient precision of semimajor axes, the theory of Asher \& Clube (1993) and Asher \& Izumi (1998) that the meteoroids responsible for enhanced Taurid activity are in 7:2 resonance with Jupiter could be confirmed (at least for 2015 meteors). This fact cannot be revealed from lower precision data such as those of Matlovič et al. (2017) $)^{3}$. Moreover, we found that the Taurid branch, which is responsible for the enhanced activity in 2015, forms an interesting orbital structure. Although the enhanced activity lasted for 23 days according to our data, all orbits had very similar orientation of the line of apsides, i.e., the longitude and latitude of perihelion. Since semimajor axes were in a narrow range and all observed meteoroids had to intersect Earth's orbit, only one free parameter remains. That is why there is a good correlation between the longitude of the ascending node (or, equivalently, solar longitude at the date of observation), eccentricity, and perihelion distance.

There was, nevertheless, some spread of orbital elements within the new branch. The longitudes of perihelia were within the range $155.9-160^{\circ}$ and latitudes of perihelia within the range $4.2-5.7^{\circ}$. The semimajor axes were within the resonance limits, 2.23-2.28 AU. The additional condition for the new branch membership follows from the limited period of activity and can

\footnotetext{
3 We observed 10 fireballs from their sample and their semimajor axes are often off with respect to ours by several tenths of AU.
}

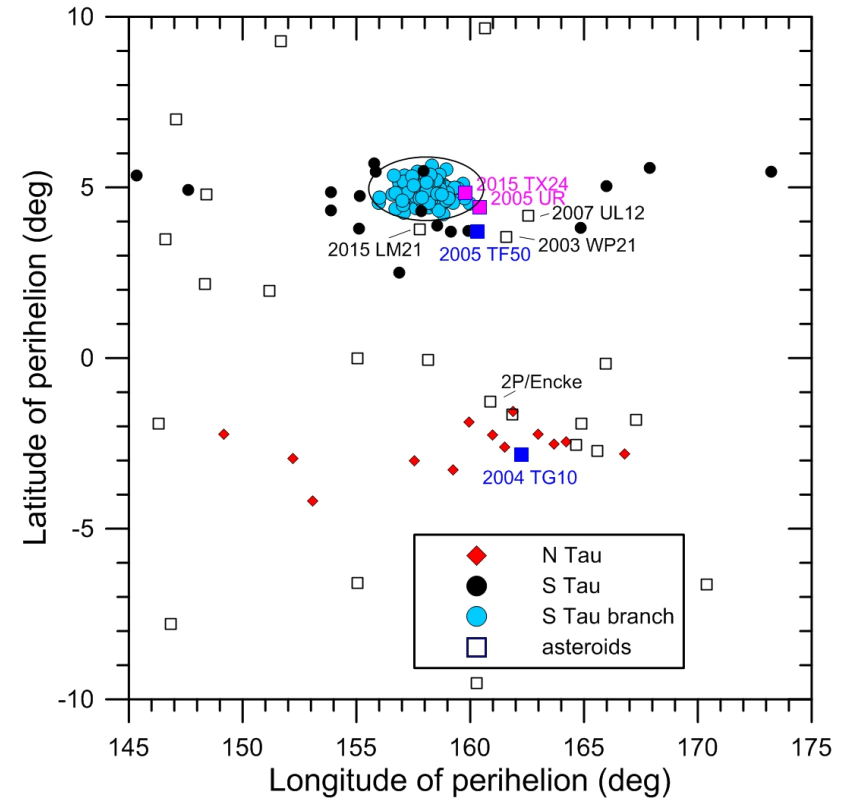

Fig. 22. Comparison of perihelia orientation of 2015 Taurids with asteroids and comets from the JPL database.

be expressed, for example, in terms of eccentricity lying between 0.80-0.90. Three asteroids, 2015 TX24, 2005 UR, and 2005 TF50, fully or nearly satisfy all these conditions. Figure 22 shows three other asteroids (2003 WP21, 2007 UL12, and 2015 LM21) with perihelia orientation not far from the new branch, but none of them simultaneously fulfills both the semimajor axis and eccentricity criteria.

Since the Earth does not encounter the new branch every year, it is evident that meteoroids of the new branch are not spread along the whole orbit. The model and observations of Asher \& Clube (1993) and Asher \& Izumi (1998) suggest that the enhanced activity of Taurids is caused by a resonant swarm of meteoroids, which extends $\pm 30-40^{\circ}$ from the center of the swarm in mean anomaly. It does not, however, necessarily mean that the new branch observed in 2015 is identical to or representative of the whole swarm. The orbits of meteoroids observed by the EN during the enhanced activity in 1995 had somewhat different characteristics than in 2015; these had larger semimajor axes and smaller perihelia, which did not change so much with solar longitude.

The new branch contains quite large bodies. Our brightest fireball was caused by a body in excess of $1000 \mathrm{~kg}$, which corresponds to diameter more than one meter, assuming that bulk density was not higher than $2000 \mathrm{~kg} \mathrm{~m}^{-3}$. This body was disintegrated very high in the atmosphere and likely had high porosity and low bulk density. The NASA JPL fireball page ${ }^{4}$ lists a fireball with 10 times higher radiated energy, which occurred on the same day (October 31, 2015 11:34:30 UT) above the Pacific Ocean at a quite large height of $71 \mathrm{~km}$. Considering the unusual height, it is likely that that fireball belonged to the Taurid new branch as well. The size of that body was $2-3 \mathrm{~m}$ or more. Two similar, slightly smaller, events occurred on November 2, 2005 (05:16:47 and 07:04:32), also over Pacific Ocean. The heights of these bolides were 74 and $68.5 \mathrm{~km}$, respectively. These three fireballs are among the top five events with largest heights among

4 http://neo.jpl.nasa.gov/fireballs/, accessed February 3, 2017. 
Table 3. Orbital elements of asteroids discussed here as taken from the JPL database and converted to J2000.0 equinox.

\begin{tabular}{llllllllll}
\hline \hline Asteroid & $\lambda_{\odot}$ & $a$ & $e$ & $q$ & $i$ & $\omega$ & $\Omega$ & $\pi$ & $\beta$ \\
\hline 2005 UR & 216.44 & 2.254 & 0.882 & 0.266 & 6.94 & 140.40 & 20.03 & 160.43 & 4.42 \\
2015 TX24 & 218.81 & 2.269 & 0.872 & 0.290 & 6.05 & 126.80 & 32.99 & 159.79 & 4.84 \\
\hline 2005 TF50 & 219.60 & 2.272 & 0.869 & 0.298 & 10.70 & 159.67 & 0.66 & 160.33 & 3.70 \\
2004 TG10 & 223.32 & 2.234 & 0.862 & 0.308 & 4.18 & 317.11 & 205.13 & 162.23 & -2.84 \\
\hline
\end{tabular}

the 288 fireballs with known heights listed at the NASA JPL page. Their trajectories and velocities are not given but the Taurid radiant was above the horizon in all cases. We note that 2005 was also a year of enhanced Taurid activity.

Fireball data therefore prove the presence of meter-sized bodies among the Taurid new branch. Based on orbital similarity, we argue that asteroids of several hundred meters in diameter are members of the Taurid new branch as well. This is almost certain for 2015 TX24, very likely for 2005 UR, and possible for 2005 TF50. We are not speaking about a distant relationship. The discovered Taurid branch is simply a population of bodies with the size range from several millimeters to several hundred of meters, which all move together around the Sun. Every few years, the Earth is encountering this branch for a period of about three weeks. During that time, the chance of impact of an asteroid of significant size (tens of meters) is significantly enhanced. Even if intrinsically weak, bodies of such size can penetrate deep in the atmosphere (Shuvalov \& Artemieva 2002) and pose a hazard to the ground.

We will allow theoretical celestial mechanicians to explain the formation and evolution of the Taurid new branch and the Taurid complex as a whole. A structure similar to the new branch could be created by a disruption of a parent body at heliocentric distance of about 3.6 AU (where the orbits come close together) but ejection velocities up to $1.5 \mathrm{~km} \mathrm{~s}^{-1}$ and subsequent removal of all non-resonant orbits would be needed. Also, asteroids 2005 TF50, 2015 TX24, and 2005 UR can all be related to 2004 TG10 but located at a different phase along the secular cycle as computed for 2004 TG10 by Porubčan et al. (2006). Asteroid 2005 TF50 is about 2000 yr behind, 2005 UR is about $2300 \mathrm{yr}$ behind, and 2015 TX24 is about $2400 \mathrm{yr}$ behind 2004 TG10. The elements $\omega, \Omega$, and $i$ all agree well with this assumption. For 2005 TF50 and 2015 TX24 $e$ and $q$ are also in agreement. The new Taurid branch can be also part of this relation. In fact, the orbital elements of the theoretical Southern Taurid meteors derived from 2004 TG10, as computed by Babadzhanov et al. (2008), fall perfectly among the Taurid branch fireballs in Fig. 12. Only in $\pi$ there is a difference of $2.5^{\circ}$. But only the central part of the new branch at $\lambda_{\odot} \sim 220^{\circ}$ can be explained in this way.

\section{Conclusions}

We presented data of unprecedented precision for a large sample of 144 Taurid fireballs observed by the European fireball network in 2015. This data set contains precise and detailed data on the Taurids covering 7 orders in mass, i.e., from tenths of a gram to one-ton meteoroids. We have shown that the enhanced Taurid activity in 2015 was produced by a well-defined branch embedded within the much broader Southern Taurid stream. The new branch can be characterized by the longitudes of perihelia lying between $155.9-160^{\circ}$, latitudes of perihelia between $4.2-$ $5.7^{\circ}$, semimajor axes between $2.23-2.28 \mathrm{AU}$, and eccentricities between $0.80-0.90$. These orbits form a concentric ring in the inner solar system with perihelia between $0.23-0.45$ AU. The new branch lies within the semimajor axis range spanned by the $7: 2$ resonance, indicating strongly that the meteoroids responsible for the outbursts are within this resonance, as expected from the model of Asher \& Clube (1993). The Earth was the encountering members of the new branch at their ascending nodes between October 25 and November 17. The orbital configuration of the branch cause meteoroids with progressively lower eccentricities, larger perihelion distances, and lower entry velocities to encounter the Earth during the activity period.

The explanation of the structure and evolution of the new branch and its relation to the whole Taurid complex must be left to future theoretical studies. Nevertheless, we confirm earlier observations that the Taurid stream contains large meteoroids. This is valid for the new branch in particular. The largest object we observed was at least one meter in diameter. A ten times more massive object observed on the same day over the Pacific Ocean probably belonged to this new branch as well. Moreover, the orbits of asteroids 2015 TX24 and 2005 UR, both of diameters of several hundreds of meters, place them within the new Taurid branch as well. It is therefore very likely that the branch also contains numerous objects of decameter size. Although our data show that large Taurids have porous and fragile structure, objects of tens or hundreds of meters in size pose a hazard to the ground even if they have low intrinsic strength. Theoretical and observational studies and searches for related asteroids belonging to this newly discovered and described branch of Southern Taurids are therefore highly recommended. A better understanding of this real source of potentially hazardous objects that are large enough to cause significant regional or even continental damage on the Earth is a task of capital importance.

Acknowledgements. This work was supported by the Praemium Academiae of the Czech Academy of Sciences, grant 16-00761S from the Czech Science Foundation, and the Czech institutional project RVO:67985815. Operation of the Slovak station Stará Lesná was supported by the project ITMS No. 26220120029, based on the supporting operational Research and development program financed from the European Regional Development Fund. We thank especially J. Keclíková, but also L. Shrbený and H. Zichová for a careful measuring of all photographic images. We also thank B. Pelc, T. Chlíbec, L. Sklenár and D. Ščerba for their images of two brightest Taurids.

\section{References}

Asher, D. J., \& Clube, S. V. M. 1993, QJRAS, 34, 481

Asher, D. J., \& Izumi, K. 1998, MNRAS, 297, 23

Asher, D. J., Clube, S. V. M., \& Steel, D. I. 1993, MNRAS, 264, 93

Babadzhanov, P. B. 2001, A\&A, 373, 329

Babadzhanov, P. B., Williams, I. P., \& Kokhirova, G. I. 2008, MNRAS, 386, 1436

Borovička, J. 1990, Bull. Astr. Inst. Czechosl., 41, 391

Borovička, J., Tóth, J., Igaz, A., et al. 2013, Meteorit. Planet. Sci., 48, 1757

Borovička, J., Spurný, P., Grigore, V. I., \& Svoreň, J., 2017, Planet. Space Sci., 143,147

Brown, P., Marchenko, V., Moser, D. E., Weryk, R., \& Cooke, W. 2013, Meteorit. Planet. Sci., 48, 270 
P. Spurný et al.: Discovery of a new branch of the Taurid meteoroid stream

Ceplecha, Z. 1987, Bull. Astr. Inst. Czechosl., 38, 222

Ceplecha, Z. 1988, Bull. Astr. Inst. Czechosl., 39, 221

Ceplecha, Z., \& McCrosky, R. E. 1976, J. Geophys. Res., 81, 6257

Ceplecha, Z., Spurný, P., Borovička, J., \& Keclíková, J. 1993, A\&A, 279, 615

Ceplecha, Z., Borovička, J., Elford, W. G., et al. 1998, Space Sci. Rev., 84, 327

Clark, D. L., \& Wiegert, P. A. 2011, Meteorit. Planet. Sci., 46, 1217

Clube, S. V. M., \& Napier, W. M. 1984, MNRAS, 211, 953

Dubietis, A., \& Arlt, R. 2007, MNRAS, 376, 890

Jenniskens, P. 2006, Meteor Showers and their Parent Comets (Cambridge Univ. Press)

Johannink, C., \& Miskotte, K. 2006, J. Int. Meteor. Organization, 34, 7

Madiedo, J. M., Ortiz, J. L., Trigo-Rodríguez, J. M., et al. 2014, Icarus, 231, 356

Matlovič, P., Tóth, J., Rudawska, R., \& Kornoš, L. 2017, Planet. Space Sci., 143 104

McBeath, A. 1999, J. Int. Meteor. Organization, 27, 53

Napier, W. M. 2010, MNRAS, 405, 1901

Nugent, C. R., Mainzer, A., Masiero, J., et al. 2015, ApJ, 814, 117
Olech, A., Źołądek, P., Wiśniewski, M., et al. 2016, MNRAS, 461, 674

Popescu, M., Birlan, M., Nedelcu, D. A., Vaubaillon, J., \& Cristescu, C. P. 2014, A\&A, 572, A106

Porubčan, V., Kornoš, L., \& Williams, I. P. 2006, Contrib. Astron. Obs. Skalnaté Pleso, 36, 103

ReVelle, D. O., \& Ceplecha, Z. 2001, in Proc. Meteoroids 2001 Conference, Kiruna, Sweden. ed. B., Warmbein, ESA SP, 495, 507

Shiba, Y. 2016, J. Int. Meteor. Organization, 44, 78

Shuvalov, V. V., \& Artemieva, N. A. 2002, Planet. Space Sci., 50, 181

Spurný, P. 1996, High fireball activity of the new subsystem of the Taurid complex, ACM 1996, Versailles, poster presentation

Spurný, P., Borovička, J., \& Shrbený, L. 2007, in Near Earth Objects, our Celestial Neighbors: Opportunity and Risk, eds. G.B. Valsecchi, D. Vokrouhlický, \& A. Milani (Cambridge University Press), Proc. IAU Symp., 236, 121

Tancredi, G. 2014, Icarus, 233, 66

Whipple, F. L. 1940, Proc. Amer. Philos. Soc., 83, 711 


\section{Appendix A: Additional tables}

Table A.1. Radiant and orbital data for 2015 Taurid fireballs.

\begin{tabular}{|c|c|c|c|c|c|c|c|c|c|c|c|}
\hline Code & Branch $^{1}$ & $\lambda_{\odot}$ & $\alpha_{\mathrm{g}}$ & $\delta_{\mathrm{g}}$ & $v_{\mathrm{g}}$ & $a$ & $e$ & $q$ & $\omega$ & $i$ & $\pi$ \\
\hline \multirow[t]{2}{*}{ EN231015_204348 } & \multirow[t]{2}{*}{$\mathrm{S}$} & \multirow[t]{2}{*}{209.908} & 49.00 & 15.31 & 35.04 & 2.387 & 0.9249 & 0.1792 & 134.92 & 5.39 & 164.84 \\
\hline & & & 0.01 & 0.02 & 0.03 & 0.012 & 0.0005 & 0.0003 & 0.03 & 0.04 & 0.03 \\
\hline \multirow[t]{2}{*}{ EN231015_211327 } & \multirow[t]{2}{*}{$\mathrm{N}$} & \multirow[t]{2}{*}{209.928} & 42.26 & 20.04 & 30.16 & 1.999 & 0.8578 & 0.2842 & 303.17 & 5.02 & 153.09 \\
\hline & & & 0.02 & 0.02 & 0.06 & 0.013 & 0.0012 & 0.0006 & 0.03 & 0.04 & 0.03 \\
\hline \multirow{2}{*}{ EN241015_004546 } & \multirow[t]{2}{*}{$\mathrm{N}$} & \multirow[t]{2}{*}{210.075} & 45.88 & 18.83 & 32.44 & 2.074 & 0.8913 & 0.2255 & 309.90 & 2.46 & 159.94 \\
\hline & & & 0.12 & 0.08 & 0.03 & 0.020 & 0.0006 & 0.0014 & 0.24 & 0.14 & 0.24 \\
\hline \multirow[t]{2}{*}{ EN241015_185031 } & \multirow[t]{2}{*}{$\mathrm{S}$} & \multirow[t]{2}{*}{210.825} & 42.91 & 11.61 & 28.19 & 1.975 & 0.8272 & 0.3414 & 116.78 & 5.51 & 147.62 \\
\hline & & & 0.01 & 0.01 & 0.01 & 0.003 & 0.0003 & 0.0002 & 0.02 & 0.01 & 0.02 \\
\hline \multirow{2}{*}{ EN251015_022301 } & SB & 211.138 & 47.62 & 13.76 & 32.63 & 2.269 & 0.8955 & 0.2370 & 127.73 & 6.26 & 158.88 \\
\hline & & & 0.01 & 0.04 & 0.05 & 0.013 & 0.0008 & 0.0005 & 0.04 & 0.06 & 0.04 \\
\hline EN251015_031725 & SB & 211.176 & 46.32 & 13.59 & 31.73 & 2.265 & 0.8847 & 0.2612 & 124.80 & 5.54 & 155.99 \\
\hline & & & 0.03 & 0.02 & 0.05 & 0.013 & 0.0008 & 0.0006 & 0.06 & 0.03 & 0.06 \\
\hline EN261015_213736 & SB & 212.933 & 48.99 & 14.44 & 32.37 & 2.253 & 0.8921 & 0.2430 & 127.03 & 5.66 & 159.97 \\
\hline & & & 0.03 & 0.03 & 0.10 & 0.028 & 0.0017 & 0.0010 & 0.07 & 0.05 & 0.07 \\
\hline EN261015_224031 & SB & 212.977 & 48.51 & 13.96 & 31.98 & 2.263 & 0.8874 & 0.2549 & 125.55 & 5.99 & 158.54 \\
\hline & & & 0.03 & 0.03 & 0.08 & 0.022 & 0.0014 & 0.0008 & 0.06 & 0.05 & 0.06 \\
\hline EN271015_220749 & SB & 213.951 & 49.26 & 13.94 & 31.74 & 2.246 & 0.8838 & 0.2610 & 124.85 & 6.19 & 158.82 \\
\hline & & & 0.01 & 0.05 & 0.03 & 0.008 & 0.0005 & 0.0004 & 0.04 & 0.07 & 0.04 \\
\hline EN281015_011855 & S & 214.084 & 43.91 & 11.12 & 26.78 & 2.005 & 0.8057 & 0.3896 & 111.24 & 5.73 & 145.34 \\
\hline & & & 0.03 & 0.07 & 0.07 & 0.014 & 0.0018 & 0.0010 & 0.08 & 0.07 & 0.08 \\
\hline EN301015_222401 & SB & 216.958 & 51.08 & 14.52 & 30.96 & 2.235 & 0.8733 & 0.2832 & 122.22 & 5.65 & 159.19 \\
\hline & & & 0.02 & 0.06 & 0.05 & 0.013 & 0.0009 & 0.0006 & 0.05 & 0.08 & 0.05 \\
\hline EN311015_002325 & $\mathrm{N}$ & 217.040 & 44.77 & 19.16 & 28.36 & 2.349 & 0.8436 & 0.3674 & 292.16 & 2.41 & 149.17 \\
\hline & & & 0.01 & 0.01 & 0.04 & 0.012 & 0.0010 & 0.0005 & 0.02 & 0.01 & 0.02 \\
\hline EN311015_023900 & SB & 217.134 & 51.15 & 14.34 & 30.90 & 2.246 & 0.8726 & 0.2861 & 121.83 & 5.89 & 158.98 \\
\hline & & & 0.01 & 0.03 & 0.04 & 0.011 & 0.0008 & 0.0005 & 0.04 & 0.04 & 0.04 \\
\hline EN311015_025717 & SB & 217.147 & 51.20 & 14.38 & 30.94 & 2.249 & 0.8732 & 0.2850 & 121.95 & 5.86 & 159.11 \\
\hline & & & 0.52 & 0.04 & 0.03 & 0.089 & 0.0024 & 0.0061 & 1.03 & 0.24 & 1.03 \\
\hline EN311015_172431 & SB & 217.749 & 51.56 & 14.50 & 30.82 & 2.259 & 0.8719 & 0.2893 & 121.40 & 5.76 & 159.17 \\
\hline & & & 0.02 & 0.03 & 0.04 & 0.012 & 0.0008 & 0.0004 & 0.04 & 0.05 & 0.04 \\
\hline EN311015_180520 & SB & 217.777 & 51.69 & 14.59 & 30.87 & 2.250 & 0.8724 & 0.2872 & 121.69 & 5.71 & 159.48 \\
\hline & & & 0.01 & 0.02 & 0.03 & 0.009 & 0.0006 & 0.0003 & 0.02 & 0.02 & 0.02 \\
\hline EN311015_182902 & SB & 217.794 & 50.83 & 14.50 & 30.37 & 2.267 & 0.8665 & 0.3026 & 119.81 & 5.31 & 157.62 \\
\hline & & & 0.02 & 0.03 & 0.07 & 0.021 & 0.0015 & 0.0007 & 0.03 & 0.03 & 0.03 \\
\hline EN311015_185530 & SB & 217.812 & 51.17 & 14.68 & 30.59 & 2.265 & 0.8693 & 0.2960 & 120.59 & 5.28 & 158.42 \\
\hline & & & 0.01 & 0.03 & 0.05 & 0.015 & 0.0011 & 0.0005 & 0.03 & 0.04 & 0.03 \\
\hline EN311015_192126 & $\mathrm{S}$ & 217.830 & 49.84 & 13.99 & 29.62 & 2.260 & 0.8563 & 0.3247 & 117.29 & 5.34 & 155.13 \\
\hline & & & 0.01 & 0.01 & 0.03 & 0.007 & 0.0006 & 0.0003 & 0.02 & 0.02 & 0.02 \\
\hline EN311015_200534 & SB & 217.861 & 51.36 & 14.57 & 30.59 & 2.249 & 0.8689 & 0.2949 & 120.78 & 5.51 & 158.65 \\
\hline & & & 0.02 & 0.07 & 0.06 & 0.017 & 0.0012 & 0.0007 & 0.06 & 0.09 & 0.06 \\
\hline EN311015_202117 & SB & 217.872 & 50.82 & 14.66 & 30.23 & 2.238 & 0.8640 & 0.3044 & 119.71 & 5.06 & 157.60 \\
\hline & & & 0.01 & 0.01 & 0.00 & 0.002 & 0.0001 & 0.0001 & 0.02 & 0.01 & 0.02 \\
\hline EN311015_211904 & SB & 217.912 & 51.80 & 14.74 & 30.96 & 2.275 & 0.8743 & 0.2860 & 121.73 & 5.56 & 159.65 \\
\hline & & & 0.06 & 0.02 & 0.06 & 0.019 & 0.0011 & 0.0009 & 0.13 & 0.04 & 0.13 \\
\hline EN311015_230919 & SB & 217.988 & 51.80 & 14.69 & 30.79 & 2.245 & 0.8713 & 0.2889 & 121.49 & 5.58 & 159.50 \\
\hline & & & 0.03 & 0.02 & 0.06 & 0.015 & 0.0011 & 0.0006 & 0.06 & 0.03 & 0.06 \\
\hline EN311015_231301 & SB & 217.991 & 51.44 & 14.49 & 30.59 & 2.258 & 0.8689 & 0.2960 & 120.62 & 5.62 & 158.62 \\
\hline & & & 0.02 & 0.04 & 0.10 & 0.027 & 0.0020 & 0.0010 & 0.06 & 0.06 & 0.06 \\
\hline EN011115_013625 & SB & 218.091 & 51.42 & 14.58 & 30.60 & 2.279 & 0.8696 & 0.2 & 120.41 & 5.49 & 158.51 \\
\hline & & & 0.01 & 0.03 & 0.05 & 0.013 & 0.0009 & 0.0 & 0.03 & 0.03 & 0.03 \\
\hline EN011115_033911 & $\mathrm{N}$ & 218.176 & 50.88 & 20.51 & 31.26 & 2.291 & 0.8793 & & 302.85 & 2.69 & 160.99 \\
\hline & & & 0.28 & 0.06 & 0.11 & 0.056 & 0.0022 & & 0.56 & 0.11 & 0.56 \\
\hline EN011115_174410 & SB & 218.763 & 51.87 & 14.84 & 30.40 & 2.256 & 0.8665 & 0.3013 & 120.00 & 5.22 & 158.77 \\
\hline & & & 0.01 & 0.02 & 0.01 & 0.004 & 0.0003 & 0.0002 & 0.02 & 0.02 & 0.02 \\
\hline EN011115_183646 & $\mathrm{S}$ & 218.799 & 51.61 & 15.34 & 30.31 & 2.259 & 0.8657 & 0.3034 & 119.74 & 4.46 & 158.55 \\
\hline & & & 0.06 & 0.06 & 0.19 & 0.055 & 0.0040 & 0.0019 & 0.13 & 0.08 & 0.13 \\
\hline EN011115_191104 & SB & 218.823 & 51.60 & 14.15 & 29.98 & 2.231 & 0.8599 & 0.3127 & 118.77 & 5.86 & 157.61 \\
\hline & & & 0.01 & 0.01 & 0.03 & 0.007 & 0.0005 & 0.0003 & 0.02 & 0.01 & 0.02 \\
\hline EN011115_200918 & SB & 218.864 & 51.96 & 14.48 & 30.28 & 2.244 & 0.8643 & 0.3044 & 119.68 & 5.68 & 158.56 \\
\hline & & & 0.03 & 0.06 & 0.04 & 0.013 & 0.0009 & 0.0006 & 0.07 & 0.08 & 0.07 \\
\hline
\end{tabular}

Notes. The code of each fireball also contains the date (in ddmmyy format) and GMT time corresponding to beginning rounded to whole second (in hhmmss format). ${ }^{(1)} \mathrm{N}$ - Northern (13), S - Southern (18), SB - Southern new branch (113) 
Table A.1. continued.

\begin{tabular}{|c|c|c|c|c|c|c|c|c|c|c|c|}
\hline Code & Branch $^{1}$ & $\lambda_{\odot}$ & $\alpha_{\mathrm{g}}$ & $\delta_{\mathrm{g}}$ & $v_{\mathrm{g}}$ & $a$ & $e$ & $q$ & $\omega$ & $i$ & $\pi$ \\
\hline \multirow[t]{2}{*}{ EN011115_223909 } & \multirow[t]{2}{*}{ SB } & \multirow{2}{*}{218.968} & 52.28 & 14.64 & 30.47 & 2.253 & 0.8671 & 0.2994 & 120.23 & 5.64 & 159.21 \\
\hline & & & 0.01 & 0.02 & 0.04 & 0.011 & 0.0008 & 0.0004 & 0.03 & 0.03 & 0.03 \\
\hline \multirow[t]{2}{*}{ EN011115_234207 } & \multirow[t]{2}{*}{ SB } & \multirow[t]{2}{*}{219.011} & 52.47 & 14.88 & 30.64 & 2.263 & 0.8697 & 0.2948 & 120.72 & 5.46 & 159.75 \\
\hline & & & 0.03 & 0.03 & 0.16 & 0.043 & 0.0032 & 0.0016 & 0.07 & 0.05 & 0.07 \\
\hline \multirow[t]{2}{*}{ EN021115_020950 } & \multirow[t]{2}{*}{ SB } & \multirow[t]{2}{*}{219.114} & 51.90 & 14.79 & 30.26 & 2.278 & 0.8652 & 0.3071 & 119.23 & 5.22 & 158.36 \\
\hline & & & 0.02 & 0.02 & 0.04 & 0.012 & 0.0009 & 0.0005 & 0.04 & 0.02 & 0.04 \\
\hline \multirow{2}{*}{ EN021115_021740 } & \multirow{2}{*}{ SB } & 219.119 & 51.96 & 14.57 & 30.19 & 2.262 & 0.8638 & 0.3081 & 119.18 & 5.51 & 158.31 \\
\hline & & & 0.02 & 0.05 & 0.05 & 0.014 & 0.0011 & 0.0007 & 0.05 & 0.06 & 0.05 \\
\hline EN021115_022525 & SB & 219.125 & 52.64 & 14.55 & 30.60 & 2.259 & 0.8688 & 0.2964 & 120.56 & 5.93 & 159.70 \\
\hline & & & 0.03 & 0.04 & 0.05 & 0.013 & 0.0009 & 0.0006 & 0.06 & 0.05 & 0.06 \\
\hline EN021115_024553 & $\mathrm{N}$ & 219.139 & 47.24 & 20.59 & 27.81 & 2.121 & 0.8269 & 0.3671 & 293.11 & 3.20 & 152.22 \\
\hline & & & 0.05 & 0.02 & 0.04 & 0.010 & 0.0009 & 0.0007 & 0.10 & 0.02 & 0.10 \\
\hline EN021115_182450 & SB & 219.792 & 51.84 & 14.14 & 29.61 & 2.262 & 0.8558 & 0.3262 & 117.09 & 5.76 & 156.90 \\
\hline & & & 0.39 & 0.19 & 0.04 & 0.064 & 0.0022 & 0.0046 & 0.77 & 0.28 & 0.77 \\
\hline EN021115_195540 & SB & 219.855 & 52.70 & 14.82 & 30.26 & 2.274 & 0.8649 & 0.3072 & 119.23 & 5.44 & 159.10 \\
\hline & & & 0.02 & 0.03 & 0.02 & 0.005 & 0.0003 & 0.0003 & 0.04 & 0.04 & 0.04 \\
\hline EN021115_201534 & SB & 219.868 & 52.22 & 14.95 & 29.97 & 2.272 & 0.8612 & 0.3153 & 118.31 & 5.01 & 158.19 \\
\hline & & & 0.03 & 0.03 & 0.09 & 0.027 & 0.0020 & 0.0010 & 0.06 & 0.04 & 0.06 \\
\hline EN021115_205431 & $\mathrm{N}$ & 219.895 & 52.44 & 20.31 & 30.88 & 2.248 & 0.8734 & 0.2845 & 302.04 & 1.84 & 161.90 \\
\hline & & & 0.02 & 0.02 & 0.05 & 0.013 & 0.0009 & 0.0005 & 0.03 & 0.03 & 0.03 \\
\hline EN021115_213614 & SB & 219.925 & 52.39 & 14.96 & 30.03 & 2.270 & 0.8620 & 0.3134 & 118.53 & 5.07 & 158.47 \\
\hline & & & 0.02 & 0.05 & 0.07 & 0.021 & 0.0015 & 0.0008 & 0.05 & 0.06 & 0.05 \\
\hline EN021115_215818 & SB & 219.940 & 52.49 & 14.71 & 30.05 & 2.272 & 0.8620 & 0.3134 & 118.52 & 5.43 & 158.48 \\
\hline & & & 0.02 & 0.07 & 0.05 & 0.014 & 0.0010 & 0.0006 & 0.06 & 0.09 & 0.06 \\
\hline EN021115_220435 & SB & 219.944 & 52.45 & 14.70 & 29.99 & 2.265 & 0.8611 & 0.3146 & 118.41 & 5.41 & 158.37 \\
\hline & & & 0.01 & 0.02 & 0.05 & 0.013 & 0.0010 & 0.0005 & 0.03 & 0.02 & 0.03 \\
\hline 232112 & SB & 219.998 & 52.14 & 14.55 & 29.78 & 2.277 & 0.8587 & 0.3217 & 117.55 & 5.41 & 157.56 \\
\hline & & & 0.01 & 0.02 & 0.04 & 0.012 & 0.0009 & 0.0005 & 0.03 & 0.03 & 0.03 \\
\hline 234348 & S & 220.013 & 50.43 & 14.32 & 28.59 & 2.247 & 0.8419 & 0.3552 & 113.84 & 4.73 & 153.87 \\
\hline & & & 0.04 & 0.06 & 0.11 & 0.029 & 0.0026 & 0.0013 & 0.09 & 0.07 & 0.09 \\
\hline EN021115_235259 & SB & 220.020 & 52.65 & 14.71 & 30.02 & 2.250 & 0.8610 & 0.3128 & 118.67 & 5.48 & 158.70 \\
\hline & & & 0.01 & 0.01 & 0.03 & 0.008 & 0.0006 & 0.0003 & 0.02 & 0.01 & 0.02 \\
\hline EN031115_002007 & SB & 220.038 & 52.51 & 14.65 & 29.83 & 2.232 & 0.8580 & 0.3169 & 118.27 & 5.44 & 158.33 \\
\hline & & & 0.02 & 0.03 & 0.03 & 0.009 & 0.0007 & 0.0004 & 0.04 & 0.04 & 0.04 \\
\hline EN031115_011247 & SB & 220.075 & 53.06 & 14.70 & 30.22 & 2.249 & 0.8636 & 0.3068 & 119.37 & 5.70 & 159.46 \\
\hline & & & 0.02 & 0.02 & 0.06 & 0.017 & 0.0013 & 0.0007 & 0.04 & 0.03 & 0.04 \\
\hline EN031115_012404 & SB & 220.083 & 52.76 & 13.98 & 30.02 & 2.278 & 0.8613 & 0.3159 & 118.21 & 6.41 & 158.31 \\
\hline & & & 0.02 & 0.05 & 0.09 & 0.023 & 0.0018 & 0.0010 & 0.06 & 0.07 & 0.06 \\
\hline EN031115_025102 & SB & 220.143 & 52.88 & 14.59 & 30.06 & 2.253 & 0.8616 & 0.3119 & 118.76 & 5.72 & 158.92 \\
\hline & & & 0.02 & 0.12 & 0.04 & 0.013 & 0.0009 & 0.0008 & 0.10 & 0.15 & 0.10 \\
\hline EN031115_031920 & SB & 220.163 & 52.25 & 14.27 & 29.67 & 2.269 & 0.8568 & 0.3250 & 117.19 & 5.74 & 157.37 \\
\hline & & & 0.02 & 0.02 & 0.12 & 0.030 & 0.0025 & 0.0014 & 0.06 & 0.03 & 0.06 \\
\hline EN031115_193751 & SB & 220.844 & 52.95 & 14.53 & 29.73 & 2.276 & 0.8577 & 0.3239 & 117.29 & 5.65 & 158.14 \\
\hline & & & 0.07 & 0.03 & 0.03 & 0.014 & 0.0007 & 0.0008 & 0.14 & 0.04 & 0.14 \\
\hline EN031115_195654 & SB & 220.857 & 53.26 & 14.89 & 29.91 & 2.261 & 0.8599 & 0.3168 & 118.16 & 5.37 & 159.03 \\
\hline & & & 0.04 & 0.08 & 0.10 & 0.029 & 0.0022 & 0.0011 & 0.09 & 0.10 & 0.09 \\
\hline EN031115_202247 & $\mathrm{N}$ & 220.875 & 51.46 & 21.69 & 29.92 & 2.262 & 0.8607 & 0.3152 & 298.38 & 3.73 & 159.24 \\
\hline & & & 0.07 & 0.06 & 0.10 & 0.030 & 0.0021 & 0.0012 & 0.13 & 0.08 & 0.13 \\
\hline EN031115_204226 & SB & 220.888 & 52.73 & 14.89 & 29.61 & 2.273 & 0.8564 & 0.3264 & 117.01 & 5.10 & 157.92 \\
\hline & & & 0.03 & 0.07 & 0.04 & 0.011 & 0.0008 & 0.0006 & 0.07 & 0.08 & 0.07 \\
\hline EN031115_212219 & SB & 220.916 & 52.99 & 14.75 & 29.75 & 2.278 & 0.8581 & 0.3231 & 117.37 & 5.40 & 158.30 \\
\hline & & & 0.04 & 0.03 & 0.09 & 0.027 & 0.0020 & 0.0010 & 0.08 & 0.04 & 0.08 \\
\hline EN031115_212455 & SB & 220.918 & 53.50 & 14.24 & 29.89 & 2.254 & 0.8588 & 0.3182 & 118.03 & 6.26 & 158.96 \\
\hline & & & 0.02 & 0.02 & 0.08 & 0.021 & 0.0016 & 0.0008 & 0.04 & 0.03 & 0.04 \\
\hline EN031115_213844 & SB & 220.928 & 52.78 & 14.81 & 29.60 & 2.272 & 0.8561 & 0.3269 & 116.95 & 5.21 & 157.90 \\
\hline & & & 0.11 & 0.03 & 0.05 & 0.023 & 0.0013 & 0.0014 & 0.21 & 0.06 & 0.21 \\
\hline EN031115_221917 & SB & 220.956 & 52.88 & 14.43 & 29.50 & 2.251 & 0.8539 & 0.3289 & 116.81 & 5.66 & 157.78 \\
\hline & & & 0.02 & 0.03 & 0.09 & 0.025 & 0.0020 & 0.0010 & 0.05 & 0.04 & 0.05 \\
\hline EN031115_221937 & SB & 220.956 & 52.99 & 14.90 & 29.71 & 2.268 & 0.8575 & 0.3232 & 117.39 & 5.20 & 158.37 \\
\hline & & & 0.01 & 0.03 & 0.03 & 0.009 & 0.0007 & 0.0004 & 0.04 & 0.04 & 0.04 \\
\hline EN031115_222446 & SB & 220.960 & 52.00 & 14.45 & 29.05 & 2.273 & 0.8487 & 0.3438 & 115.02 & 5.19 & 156.00 \\
\hline & & & 0.04 & 0.02 & 0.08 & 0.022 & 0.0017 & 0.0009 & 0.07 & 0.03 & 0.07 \\
\hline EN031115_225609 & SB & 220.981 & 53.44 & 14.98 & 30.02 & 2.277 & 0.8618 & 0.3148 & 118.32 & 5.36 & 159.32 \\
\hline & & & 0.02 & 0.07 & 0.05 & 0.015 & 0.0011 & 0.0007 & 0.06 & 0.09 & 0.06 \\
\hline
\end{tabular}


Table A.1. continued.

\begin{tabular}{|c|c|c|c|c|c|c|c|c|c|c|c|}
\hline Code & Branch $^{1}$ & $\lambda_{\odot}$ & $\alpha_{\mathrm{g}}$ & $\delta_{\mathrm{g}}$ & $v_{\mathrm{g}}$ & $a$ & $e$ & $q$ & $\omega$ & $i$ & $\pi$ \\
\hline \multirow[t]{2}{*}{ EN031115_230149 } & \multirow[t]{2}{*}{ SB } & \multirow[t]{2}{*}{220.985} & 53.14 & 14.53 & 29.72 & 2.269 & 0.8574 & 0.3235 & 117.36 & 5.70 & 158.36 \\
\hline & & & 0.03 & 0.02 & 0.09 & 0.026 & 0.0020 & 0.0010 & 0.06 & 0.03 & 0.06 \\
\hline \multirow{2}{*}{ EN031115_232829 } & \multirow[t]{2}{*}{ SB } & \multirow[t]{2}{*}{221.004} & 53.09 & 15.30 & 29.83 & 2.275 & 0.8595 & 0.3196 & 117.78 & 4.77 & 158.80 \\
\hline & & & 0.01 & 0.02 & 0.05 & 0.013 & 0.0010 & 0.0005 & 0.02 & 0.02 & 0.02 \\
\hline \multirow[t]{2}{*}{ EN031115_235911 } & \multirow[t]{2}{*}{ S } & \multirow[t]{2}{*}{221.025} & 51.85 & 16.41 & 28.35 & 2.058 & 0.8324 & 0.3449 & 115.81 & 2.78 & 156.87 \\
\hline & & & 0.13 & 0.18 & 0.13 & 0.032 & 0.0030 & 0.0022 & 0.27 & 0.21 & 0.27 \\
\hline \multirow{2}{*}{ EN041115_012728 } & \multirow[t]{2}{*}{ SB } & 221.087 & 52.76 & 14.08 & 29.26 & 2.245 & 0.8503 & 0.3360 & 116.01 & 5.95 & 157.12 \\
\hline & & & 0.02 & 0.01 & 0.03 & 0.009 & 0.0008 & 0.0004 & 0.04 & 0.02 & 0.04 \\
\hline EN041115_020201 & SB & 221.111 & 52.55 & 14.48 & 29.24 & 2.260 & 0.8508 & 0.3372 & 115.83 & 5.40 & 156.95 \\
\hline & & & 0.01 & 0.02 & 0.03 & 0.008 & 0.0006 & 0.0004 & 0.03 & 0.02 & 0.03 \\
\hline EN041115_021111 & SB & 221.117 & 53.60 & 14.77 & 29.98 & 2.274 & 0.8610 & 0.3160 & 118.20 & 5.65 & 159.33 \\
\hline & & & 0.05 & 0.03 & 0.04 & 0.012 & 0.0008 & 0.0007 & 0.09 & 0.05 & 0.09 \\
\hline EN041115_021452 & SB & 221.120 & 53.16 & 14.64 & 29.67 & 2.268 & 0.8568 & 0.3250 & 117.19 & 5.55 & 158.33 \\
\hline & & & 0.01 & 0.01 & 0.02 & 0.005 & 0.0003 & 0.0002 & 0.02 & 0.02 & 0.02 \\
\hline EN041115 043317 & SB & 221.216 & 53.10 & 14.11 & 29.48 & 2.269 & 0.8539 & 0.3315 & 116.44 & 6.10 & 157.67 \\
\hline & & & 0.02 & 0.02 & 0.04 & 0.010 & 0.0008 & 0.0005 & 0.04 & 0.03 & 0.04 \\
\hline EN041115_044559 & SB & 221.225 & 53.31 & 14.43 & 29.66 & 2.269 & 0.8564 & 0.3258 & 117.10 & 5.85 & 158.34 \\
\hline & & & 0.03 & 0.02 & 0.04 & 0.010 & 0.0008 & 0.0005 & 0.06 & 0.02 & 0.06 \\
\hline EN041115_203853 & SB & 221.888 & 54.05 & 14.80 & 29.71 & 2.260 & 0.8569 & 0.3234 & 117.40 & 5.64 & 159.30 \\
\hline & & & 0.02 & 0.05 & 0.05 & 0.015 & 0.0012 & 0.0006 & 0.06 & 0.06 & 0.06 \\
\hline EN041115_210403 & SB & 221.905 & 53.92 & 15.16 & 29.64 & 2.250 & 0.8559 & 0.3242 & 117.34 & 5.13 & 159.26 \\
\hline & & & 0.03 & 0.11 & 0.07 & 0.021 & 0.0016 & 0.0009 & 0.10 & 0.13 & 0.10 \\
\hline EN041115_214032 & SB & 221.931 & 53.58 & 14.86 & 29.43 & 2.267 & 0.8535 & 0.3321 & 116.37 & 5.30 & 158.32 \\
\hline & & & 0.03 & 0.02 & 0.07 & 0.020 & 0.0016 & 0.0008 & 0.06 & 0.03 & 0.06 \\
\hline EN041115_215226 & SB & 221.939 & 53.82 & 14.86 & 29.44 & 2.233 & 0.8526 & 0.3292 & 116.83 & 5.40 & 158.79 \\
\hline & & & 0.01 & 0.01 & 0.04 & 0.009 & 0.0008 & 0.0004 & 0.03 & 0.02 & 0.03 \\
\hline EN041115_225243 & SB & 221.981 & 53.66 & 14.61 & 29.37 & 2.258 & 0.8523 & 0.3335 & 116.24 & 5.61 & 158.24 \\
\hline & & & 0.02 & 0.03 & 0.10 & 0.028 & 0.0023 & 0.0011 & 0.05 & 0.04 & 0.05 \\
\hline EN041115_231355 & SB & 221.996 & 53.95 & 14.39 & 29.51 & 2.261 & 0.8541 & 0.3300 & 116.64 & 6.02 & 158.64 \\
\hline & & & 0.03 & 0.05 & 0.08 & 0.022 & 0.0018 & 0.0009 & 0.07 & 0.06 & 0.07 \\
\hline EN051115_023102 & SB & 222.133 & 53.96 & 14.96 & 29.45 & 2.241 & 0.8530 & 0.3295 & 116.77 & 5.32 & 158.91 \\
\hline & & & 0.01 & 0.03 & 0.02 & 0.006 & 0.0005 & 0.0003 & 0.03 & 0.03 & 0.03 \\
\hline EN051115_183559 & S & 222.804 & 53.24 & 13.90 & 28.48 & 2.259 & 0.8398 & 0.3618 & 113.02 & 5.93 & 155.84 \\
\hline & & & 0.01 & 0.02 & 0.01 & 0.004 & 0.0004 & 0.0002 & 0.02 & 0.02 & 0.02 \\
\hline EN051115_185259 & SB & 222.816 & 54.30 & 14.56 & 29.26 & 2.270 & 0.8510 & 0.3382 & 115.65 & 5.79 & 158.49 \\
\hline & & & 0.03 & 0.03 & 0.12 & 0.035 & 0.0028 & 0.0012 & 0.05 & 0.03 & 0.05 \\
\hline EN051115_190203 & SB & 222.823 & 54.34 & 14.66 & 29.30 & 2.270 & 0.8516 & 0.3371 & 115.78 & 5.70 & 158.62 \\
\hline & & & 0.02 & 0.02 & 0.03 & 0.010 & 0.0008 & 0.0004 & 0.03 & 0.02 & 0.03 \\
\hline EN051115_203651 & SB & 222.889 & 54.18 & 14.44 & 29.11 & 2.266 & 0.8487 & 0.3427 & 115.15 & 5.83 & 158.06 \\
\hline & & & 0.03 & 0.03 & 0.05 & 0.013 & 0.0010 & 0.0006 & 0.06 & 0.03 & 0.06 \\
\hline EN051115_205304 & $\mathrm{N}$ & 222.900 & 53.87 & 21.71 & 29.26 & 2.076 & 0.8461 & 0.3195 & 298.63 & 2.98 & 161.50 \\
\hline & & & 0.02 & 0.01 & 0.02 & 0.005 & 0.0004 & 0.0003 & 0.04 & 0.01 & 0.04 \\
\hline EN051115_212802 & SB & 222.924 & 54.21 & 14.94 & 29.06 & 2.235 & 0.8475 & 0.3409 & 115.48 & 5.26 & 158.42 \\
\hline & & & 0.02 & 0.04 & 0.04 & 0.011 & 0.0009 & 0.0005 & 0.05 & 0.04 & 0.05 \\
\hline EN051115_213128 & SB & 222.927 & 54.20 & 14.90 & 29.14 & 2.259 & 0.8492 & 0.3405 & 115.43 & 5.31 & 158.38 \\
\hline & & & 0.09 & 0.16 & 0.11 & 0.033 & 0.0024 & 0.0017 & 0.21 & 0.19 & 0.21 \\
\hline EN051115_213433 & SB & 222.929 & 53.79 & 14.60 & 28.83 & 2.255 & 0.8448 & 0.3499 & 114.38 & 5.43 & 157.32 \\
\hline & & & 0.01 & 0.02 & 0.03 & 0.007 & 0.0006 & 0.0003 & 0.02 & 0.02 & 0.02 \\
\hline EN051115_220108 & SB & 222.947 & 53.92 & 14.47 & 28.88 & 2.257 & 0.8455 & 0.3487 & 114.50 & 5.64 & 157.47 \\
\hline & & & 0.01 & 0.01 & 0.02 & 0.007 & 0.0006 & 0.0003 & 0.02 & 0.02 & 0.02 \\
\hline EN051115_221253 & SB & 222.956 & 54.30 & 14.77 & 29.13 & 2.252 & 0.8488 & 0.3404 & 115.47 & 5.50 & 158.44 \\
\hline & & & 0.05 & 0.02 & 0.03 & 0.011 & 0.0006 & 0.0007 & 0.11 & 0.03 & 0.11 \\
\hline EN051115_221501 & $\mathrm{N}$ & 222.957 & 51.92 & 21.71 & 28.80 & 2.253 & 0.8454 & 0.3484 & 294.60 & 3.32 & 157.53 \\
\hline & & & 0.01 & 0.01 & 0.08 & 0.021 & 0.0018 & 0.0008 & 0.02 & 0.02 & 0.02 \\
\hline EN051115_221906 & SB & 222.960 & 54.28 & 15.20 & 29.16 & 2.246 & 0.8493 & 0.3384 & 115.72 & 5.00 & 158.70 \\
\hline & & & 0.03 & 0.02 & 0.05 & 0.014 & 0.0012 & 0.0006 & 0.06 & 0.03 & 0.06 \\
\hline EN051115_225625 & SB & 222.986 & 54.09 & 14.54 & 28.99 & 2.262 & 0.8471 & 0.3458 & 114.81 & 5.65 & 157.81 \\
\hline & & & 0.01 & 0.04 & 0.03 & 0.008 & 0.0006 & 0.0004 & 0.04 & 0.05 & 0.04 \\
\hline EN051115_225852 & S & 222.988 & 53.90 & 15.19 & 28.53 & 2.150 & 0.8373 & 0.3498 & 114.83 & 4.74 & 157.84 \\
\hline & & & 0.01 & 0.01 & 0.04 & 0.010 & 0.0010 & 0.0005 & 0.02 & 0.01 & 0.02 \\
\hline EN051115_231201 & SB & 222.997 & 54.25 & 15.06 & 29.15 & 2.260 & 0.8495 & 0.3400 & 115.48 & 5.15 & 158.49 \\
\hline & & & 0.01 & 0.00 & 0.04 & 0.010 & 0.0009 & 0.0004 & 0.02 & 0.01 & 0.02 \\
\hline EN051115_232719 & $\mathrm{N}$ & 223.007 & 55.29 & 21.85 & 29.42 & 1.944 & 0.8445 & 0.3023 & 301.24 & 2.86 & 164.22 \\
\hline & & & 0.04 & 0.03 & 0.00 & 0.005 & 0.0001 & 0.0005 & 0.08 & 0.05 & 0.08 \\
\hline
\end{tabular}


Table A.1. continued.

\begin{tabular}{|c|c|c|c|c|c|c|c|c|c|c|c|}
\hline Code & Branch $^{1}$ & $\lambda_{\odot}$ & $\alpha_{\mathrm{g}}$ & $\delta_{\mathrm{g}}$ & $v_{\mathrm{g}}$ & $a$ & $e$ & $q$ & $\omega$ & $i$ & $\pi$ \\
\hline \multirow[t]{2}{*}{ EN051115_234939 } & \multirow[t]{2}{*}{ SB } & \multirow[t]{2}{*}{223.023} & 54.18 & 14.47 & 29.01 & 2.263 & 0.8473 & 0.3454 & 114.86 & 5.76 & 157.90 \\
\hline & & & 0.01 & 0.01 & 0.05 & 0.012 & 0.0010 & 0.0005 & 0.02 & 0.01 & 0.02 \\
\hline \multirow[t]{2}{*}{ EN051115_235119 } & \multirow[t]{2}{*}{ SB } & \multirow[t]{2}{*}{223.024} & 54.53 & 14.98 & 29.35 & 2.274 & 0.8525 & 0.3353 & 115.96 & 5.39 & 159.00 \\
\hline & & & 0.01 & 0.01 & 0.08 & 0.023 & 0.0018 & 0.0009 & 0.02 & 0.02 & 0.02 \\
\hline \multirow[t]{2}{*}{ EN061115_001740 } & \multirow[t]{2}{*}{ SB } & \multirow[t]{2}{*}{223.042} & 54.44 & 14.66 & 29.23 & 2.275 & 0.8508 & 0.3394 & 115.49 & 5.70 & 158.55 \\
\hline & & & 0.01 & 0.01 & 0.03 & 0.007 & 0.0006 & 0.0003 & 0.01 & 0.01 & 0.01 \\
\hline \multirow{2}{*}{ EN061115_002202 } & \multirow[t]{2}{*}{ S } & \multirow[t]{2}{*}{223.045} & 52.54 & 15.27 & 28.14 & 2.261 & 0.8362 & 0.3704 & 112.04 & 4.10 & 155.11 \\
\hline & & & 0.02 & 0.02 & 0.09 & 0.025 & 0.0022 & 0.0011 & 0.05 & 0.02 & 0.05 \\
\hline EN061115_003508 & SB & 223.055 & 54.24 & 15.36 & 29.15 & 2.256 & 0.8495 & 0.3395 & 115.55 & 4.79 & 158.62 \\
\hline & & & 0.03 & 0.05 & 0.07 & 0.018 & 0.0014 & 0.0008 & 0.07 & 0.06 & 0.07 \\
\hline EN061115_005009 & S & 223.065 & 54.31 & 15.97 & 29.26 & 2.251 & 0.8511 & 0.3350 & 116.08 & 4.13 & 159.16 \\
\hline & & & 0.04 & 0.13 & 0.12 & 0.033 & 0.0027 & 0.0015 & 0.12 & 0.15 & 0.12 \\
\hline EN061115_011233 & SB & 223.081 & 54.27 & 14.94 & 29.08 & 2.256 & 0.8484 & 0.3420 & 115.27 & 5.27 & 158.36 \\
\hline & & & 0.01 & 0.02 & 0.04 & 0.010 & 0.0008 & 0.0004 & 0.03 & 0.03 & 0.03 \\
\hline EN061115_011441 & SB & 223.082 & 53.57 & 15.17 & 28.72 & 2.263 & 0.8440 & 0.3530 & 113.99 & 4.67 & 157.09 \\
\hline & & & 0.09 & 0.05 & 0.10 & 0.029 & 0.0022 & 0.0015 & 0.19 & 0.06 & 0.19 \\
\hline EN061115_011623 & SB & 223.083 & 54.04 & 14.54 & 28.91 & 2.266 & 0.8462 & 0.3483 & 114.51 & 5.59 & 157.61 \\
\hline & & & 0.02 & 0.03 & 0.12 & 0.032 & 0.0027 & 0.0014 & 0.06 & 0.03 & 0.06 \\
\hline EN061115_025156 & SB & 223.150 & 54.42 & 14.65 & 29.17 & 2.279 & 0.8501 & 0.3417 & 115.22 & 5.67 & 158.38 \\
\hline & & & 0.22 & 0.03 & 0.08 & 0.041 & 0.0021 & 0.0027 & 0.43 & 0.09 & 0.43 \\
\hline EN061115_030548 & S & 223.160 & 54.69 & 16.07 & 29.54 & 2.276 & 0.8557 & 0.3284 & 116.74 & 4.18 & 159.92 \\
\hline & & & 0.02 & 0.03 & 0.05 & 0.014 & 0.0011 & 0.0007 & 0.05 & 0.04 & 0.05 \\
\hline EN061115_040629 & SB & 223.202 & 54.36 & 14.47 & 29.01 & 2.263 & 0.8473 & 0.3456 & 114.83 & 5.80 & 158.05 \\
\hline & & & 0.01 & 0.01 & 0.01 & 0.004 & 0.0003 & 0.0002 & 0.03 & 0.01 & 0.03 \\
\hline EN061115_164758 & SB & 223.732 & 54.85 & 14.88 & 28.94 & 2.240 & 0.8458 & 0.3455 & 114.93 & 5.45 & 158.68 \\
\hline & & & 0.00 & 0.01 & 0.01 & 0.002 & 0.0002 & 0.0001 & 0.01 & 0.01 & 0.01 \\
\hline EN061115_174311 & SB & 223.771 & 54.88 & 15.22 & 29.14 & 2.279 & 0.8499 & 0.3421 & 115.16 & 5.11 & 158.95 \\
\hline & & & 0.01 & 0.01 & 0.04 & 0.012 & 0.0 & 0.0004 & 0.03 & 0.02 & 0.03 \\
\hline EN071115_015331 & SB & 224.112 & 54.80 & 14.66 & 28.71 & 2.260 & 0.8432 & 0.3543 & 113.84 & 5.58 & 157.97 \\
\hline & & & 0.04 & 0.02 & 0.12 & 0.030 & 0.0027 & 0.0014 & 0.09 & 0.03 & 0.09 \\
\hline EN081115_010613 & SB & 225.083 & 55.57 & 14.79 & 28.62 & 2.271 & 0.8422 & 0.3584 & 113.33 & 5.58 & 158.43 \\
\hline & & & 0.04 & 0.03 & 0.12 & 0.032 & 0.0028 & 0.0014 & 0.08 & 0.04 & 0.08 \\
\hline EN081115_033341 & SB & 225.186 & 55.71 & 14.71 & 28.57 & 2.257 & 0.8410 & 0.3589 & 113.33 & 5.69 & 158.53 \\
\hline & & & 0.04 & 0.06 & 0.11 & 0.028 & 0.0025 & 0.0014 & 0.10 & 0.07 & 0.10 \\
\hline EN081115_181258 & SB & 225.799 & 55.87 & 14.75 & 28.31 & 2.264 & 0.8377 & 0.3674 & 112.34 & 5.58 & 158.15 \\
\hline & & & 0.01 & 0.02 & 0.01 & 0.003 & 0.0003 & 0.0002 & 0.02 & 0.02 & 0.02 \\
\hline EN081115_202907 & SB & 225.894 & 55.47 & 14.56 & 28.02 & 2.274 & 0.8341 & 0.3774 & 111.17 & 5.55 & 157.08 \\
\hline & & & 0.02 & 0.02 & 0.07 & 0.021 & 0.0018 & 0.0008 & 0.04 & 0.02 & 0.04 \\
\hline EN081115_212839 & SB & 225.935 & 55.15 & 14.83 & 27.80 & 2.260 & 0.8307 & 0.3827 & 110.63 & 5.12 & 156.58 \\
\hline & & & 0.01 & 0.01 & 0.02 & 0.007 & 0.0006 & 0.0003 & 0.01 & 0.01 & 0.01 \\
\hline EN081115_234417 & SB & 226.030 & 55.75 & 14.48 & 28.02 & 2.254 & 0.8331 & 0.3761 & 111.39 & 5.72 & 157.43 \\
\hline & & & 0.03 & 0.02 & 0.08 & 0.021 & 0.0019 & 0.0009 & 0.05 & 0.02 & 0.05 \\
\hline EN091115_001801 & SB & 226.053 & 55.15 & 15.25 & 27.82 & 2.272 & 0.8318 & 0.3822 & 110.63 & 4.67 & 156.71 \\
\hline & & & 0.02 & 0.03 & 0.16 & 0.041 & 0.0038 & 0.0017 & 0.06 & 0.03 & 0.06 \\
\hline EN091115_003545 & SB & 226.066 & 55.43 & 15.02 & 27.91 & 2.261 & 0.8323 & 0.3791 & 111.02 & 5.01 & 157.11 \\
\hline & & & 0.05 & 0.02 & 0.03 & 0.011 & 0.0008 & 0.0007 & 0.09 & 0.03 & 0.09 \\
\hline EN091115_011246 & SB & 226.092 & 55.46 & 14.33 & 27.72 & 2.237 & 0.8282 & 0.3842 & 110.55 & 5.71 & 156.66 \\
\hline & & & 0.04 & 0.10 & 0.11 & 0.028 & 0.0026 & 0.0014 & 0.11 & 0.10 & 0.11 \\
\hline EN091115_011650 & SB & 226.094 & 55.36 & 15.19 & 27.87 & 2.258 & 0.8318 & 0.3799 & 110.94 & 4.80 & 157.06 \\
\hline & & & 0.02 & 0.03 & 0.12 & 0.029 & 0.0028 & 0.0013 & 0.04 & 0.04 & 0.04 \\
\hline EN091115_032502 & SB & 226.184 & 55.99 & 15.27 & 28.27 & 2.277 & 0.8379 & 0.3691 & 112.08 & 5.00 & 158.28 \\
\hline & & & 0.02 & 0.02 & 0.02 & 0.007 & 0.0006 & 0.0004 & 0.04 & 0.03 & 0.04 \\
\hline EN091115_041944 & $\mathrm{N}$ & 226.222 & 56.51 & 21.89 & 29.90 & 2.387 & 0.8639 & 0.3248 & 296.79 & 2.51 & 162.97 \\
\hline & & & 0.03 & 0.02 & 0.06 & 0.017 & 0.0012 & 0.0007 & 0.05 & 0.03 & 0.05 \\
\hline EN101115_212402 & SB & 227.942 & 56.75 & 15.16 & 27.47 & 2.242 & 0.8252 & 0.3918 & 109.66 & 5.03 & 157.62 \\
\hline & & & 0.03 & 0.03 & 0.05 & 0.015 & 0.0014 & 0.0007 & 0.07 & 0.03 & 0.07 \\
\hline EN101115_235401 & SB & 228.047 & 57.00 & 15.45 & 27.66 & 2.260 & 0.8287 & 0.3870 & 110.11 & 4.84 & 158.18 \\
\hline & & & 0.04 & 0.02 & 0.03 & 0.009 & 0.0007 & 0.0006 & 0.08 & 0.03 & 0.08 \\
\hline EN111115_004713 & S & 228.084 & 56.33 & 13.94 & 27.01 & 2.256 & 0.8187 & 0.4090 & 107.68 & 5.99 & 155.77 \\
\hline & & & 0.01 & 0.02 & 0.08 & 0.020 & 0.0020 & 0.0009 & 0.03 & 0.02 & 0.03 \\
\hline EN111115_031037 & SB & 228.184 & 57.37 & 14.70 & 27.64 & 2.248 & 0.8275 & 0.3880 & 110.06 & 5.71 & 158.26 \\
\hline & & & 0.12 & 0.23 & 0.06 & 0.026 & 0.0017 & 0.0019 & 0.29 & 0.24 & 0.29 \\
\hline
\end{tabular}


Table A.1. continued.

\begin{tabular}{|c|c|c|c|c|c|c|c|c|c|c|c|}
\hline Code & Branch $^{1}$ & $\lambda_{\odot}$ & $\alpha_{\mathrm{g}}$ & $\delta_{\mathrm{g}}$ & $v_{\mathrm{g}}$ & $a$ & $e$ & $q$ & $\omega$ & $i$ & $\pi$ \\
\hline \multirow{2}{*}{ EN111115_181413 } & SB & 228.815 & 57.27 & 14.96 & 27.30 & 2.266 & 0.8236 & 0.3997 & 108.66 & 5.27 & 157.50 \\
\hline & & & 0.06 & 0.07 & 0.08 & 0.025 & 0.0021 & 0.0011 & 0.13 & 0.07 & 0.13 \\
\hline \multirow{2}{*}{ EN111115_181509 } & SB & 228.815 & 57.11 & 15.06 & 27.20 & 2.262 & 0.8223 & 0.4021 & 108.41 & 5.10 & 157.24 \\
\hline & & & 0.03 & 0.03 & 0.00 & 0.004 & 0.0002 & 0.0003 & 0.05 & 0.03 & 0.05 \\
\hline \multirow{2}{*}{ EN111115_184540 } & $\mathrm{N}$ & 228.837 & 58.35 & 22.60 & 29.04 & 2.286 & 0.8494 & 0.3443 & 294.88 & 2.78 & 163.68 \\
\hline & & & 0.13 & 0.02 & 0.06 & 0.026 & 0.0015 & 0.0016 & 0.25 & 0.03 & 0.25 \\
\hline \multirow[t]{2}{*}{ EN111115_203917 } & SB & 228.916 & 56.93 & 14.97 & 27.08 & 2.277 & 0.8212 & 0.4072 & 107.77 & 5.09 & 156.71 \\
\hline & & & 0.12 & 0.04 & 0.05 & 0.023 & 0.0015 & 0.0015 & 0.24 & 0.05 & 0.24 \\
\hline \multirow[t]{2}{*}{ EN111115_233243 } & SB & 229.037 & 57.63 & 15.13 & 27.34 & 2.250 & 0.8235 & 0.3970 & 109.02 & 5.19 & 158.08 \\
\hline & & & 0.03 & 0.01 & 0.04 & 0.012 & 0.0011 & 0.0006 & 0.06 & 0.02 & 0.06 \\
\hline \multirow[t]{2}{*}{ EN121115_004717 } & SB & 229.089 & 57.10 & 15.23 & 26.97 & 2.242 & 0.8183 & 0.4074 & 107.90 & 4.85 & 157.01 \\
\hline & & & 0.03 & 0.04 & 0.07 & 0.017 & 0.0017 & 0.0008 & 0.07 & 0.04 & 0.07 \\
\hline \multirow[t]{2}{*}{ EN121115_232341 } & S & 230.037 & 56.31 & 14.51 & 25.93 & 2.262 & 0.8041 & 0.4431 & 103.82 & 5.00 & 153.88 \\
\hline & & & 0.02 & 0.05 & 0.05 & 0.014 & 0.0 & 0.0007 & 0.05 & 0.04 & 0.05 \\
\hline \multirow[t]{2}{*}{ EN131115_002058 } & SB & 230.077 & 58.58 & 15.58 & 27.39 & 2.269 & 0.8251 & 0.3968 & 108.96 & 4.94 & 159.05 \\
\hline & & & 0.02 & 0.06 & 0.09 & 0.023 & 0.0022 & 0.0011 & 0.06 & 0.06 & 0.06 \\
\hline \multirow[t]{2}{*}{ EN131115_004858 } & SB & 230.097 & 58.41 & 15.35 & 27.27 & 2.275 & 0.8236 & 0.4013 & 108.42 & 5.09 & 158.54 \\
\hline & & & 0.02 & 0.02 & 0.09 & 0.023 & 22 & 0.0011 & 0.05 & 0.02 & 0.05 \\
\hline \multirow[t]{2}{*}{ EN131115_015008 } & SB & 230.139 & 58.33 & 14.98 & 27.15 & 2.277 & 0.8219 & 0.4057 & 107.93 & 5.41 & 158.09 \\
\hline & & & 0.01 & 0.01 & 0.03 & 0.009 & 0.0008 & 0.0004 & 0.03 & 0.01 & 0.03 \\
\hline \multirow[t]{2}{*}{ EN131115_042559 } & SB & 230.248 & 58.11 & 15.37 & 26.98 & 2.270 & 0.8195 & 0.4097 & 107.51 & 4.91 & 157.77 \\
\hline & & & 0.09 & 0.02 & 0.08 & 0.024 & 21 & 0.0015 & 0.17 & 0.03 & 0.17 \\
\hline \multirow[t]{2}{*}{ EN161115_193458 } & S & 233.906 & 60.56 & 14.63 & 25.65 & 2.162 & 0.7942 & 0.4450 & 104.03 & 5.66 & 157.95 \\
\hline & & & 0.03 & 0.01 & 0.02 & 0.007 & 0.0007 & 0.0004 & 0.06 & 0.01 & 0.06 \\
\hline \multirow[t]{2}{*}{ EN161115_213048 } & SB & 233.987 & 60.23 & 15.09 & 25.86 & 2.257 & 0.8025 & 0.4457 & 103.50 & 5.20 & 157.51 \\
\hline & & & 0.01 & 0.01 & 0.04 & 0.009 & 0.0010 & 0.0004 & 0.02 & 0.01 & 0.02 \\
\hline \multirow[t]{2}{*}{ EN161115_222246 } & S & 234.023 & 67.52 & 16.82 & 29.10 & 1.957 & 0.8372 & 0.3185 & 119.17 & 6.26 & 173.21 \\
\hline & & & 0.06 & 0.02 & 0.09 & 0.018 & 0.0019 & 0.0011 & 0.12 & 0.03 & 0.12 \\
\hline \multirow[t]{2}{*}{ EN171115_020907 } & SB & 234.182 & 60.80 & 15.58 & 26.20 & 2.273 & 0.8083 & 0.4357 & 104.54 & 4.94 & 158.74 \\
\hline & & & 0.02 & 0.08 & 0.00 & 0.004 & 0.0001 & 0.0004 & 0.06 & 0.08 & 0.06 \\
\hline \multirow[t]{2}{*}{ EN171115_022102 } & SB & 234.190 & 60.40 & 15.51 & 25.91 & 2.260 & 0.8036 & 0.4440 & 103.68 & 4.84 & 157.89 \\
\hline & & & 0.02 & 0.02 & 0.04 & 0.010 & 0.0010 & 0.0005 & 0.03 & 0.02 & 0.03 \\
\hline \multirow[t]{2}{*}{ EN231115_012005 } & $\mathrm{N}$ & 240.202 & 66.46 & 24.65 & 26.27 & 2.147 & 0.8035 & 0.4219 & 286.63 & 2.93 & 166.80 \\
\hline & & & 0.03 & 0.01 & 0.03 & 0.009 & 0.0009 & 0.0005 & 0.06 & 0.01 & 0.06 \\
\hline \multirow{2}{*}{ EN231115_224311 } & S & 241.102 & 69.19 & 16.03 & 25.73 & 1.992 & 0.7853 & 0.4277 & 106.76 & 5.83 & 167.88 \\
\hline & & & 0.16 & 0.02 & 0.06 & 0.022 & 0.0019 & 0.0020 & 0.31 & 0.04 & 0.31 \\
\hline \multirow{2}{*}{ EN281115_195251 } & S & 246.038 & 70.87 & 16.55 & 25.18 & 2.300 & 0.7936 & 0.4747 & 99.91 & 5.12 & 165.97 \\
\hline & & & 0.03 & 0.02 & 0.05 & 0.016 & 0.0016 & 0.0006 & 0.05 & 0.02 & 0.05 \\
\hline
\end{tabular}


P. Spurný et al.: Discovery of a new branch of the Taurid meteoroid stream

Table A.2. Physical data of 2015 Taurid fireballs.

\begin{tabular}{|c|c|c|c|c|c|c|c|c|c|c|}
\hline Code & Branch & $\begin{array}{r}v_{\infty} \\
\mathrm{km} \mathrm{s}^{-1}\end{array}$ & $\begin{array}{r}H_{\mathrm{beg}} \\
\mathrm{km}\end{array}$ & $\begin{array}{r}H_{\max } \\
\mathrm{km}\end{array}$ & $\begin{array}{r}H_{\text {end }} \\
\mathrm{km}\end{array}$ & $\begin{array}{l}Z_{\mathrm{rad}} \\
\text { deg }\end{array}$ & $\begin{array}{r}\text { Mass } \\
\mathrm{kg}\end{array}$ & Mag & $\mathrm{PE}$ & Type \\
\hline EN231015_204348 & $\mathrm{S}$ & 36.98 & 106.3 & 73.2 & 59.7 & 54.7 & 0.0055 & -7.5 & -4.52 & I \\
\hline EN231015_211327 & $\mathrm{N}$ & 32.30 & 96.9 & 64.9 & 57.8 & 38.2 & 0.0021 & -4.2 & -4.51 & I \\
\hline EN241015_004546 & $\mathrm{N}$ & 34.25 & 103.9 & 65.7 & 64.8 & 31.4 & 0.0022 & -8.2 & -4.89 & II \\
\hline EN241015_185031 & S & 30.58 & 98.8 & 73.9 & 65.6 & 67.3 & 0.012 & -6.3 & -4.87 & II \\
\hline EN251015_022301 & SB & 34.30 & 104.5 & 77.6 & 69.7 & 44.6 & 0.18 & -12.0 & -5.87 & IIIB \\
\hline EN251015_031725 & SB & 33.40 & 108.3 & 71.1 & 66.9 & 52.3 & 0.0011 & -4.9 & -4.71 & II \\
\hline EN261015_213736 & SB & 34.38 & 91.9 & 72.2 & 64.0 & 43.4 & 0.0048 & -6.4 & -4.90 & II \\
\hline EN261015_224031 & SB & 33.95 & 94.6 & 72.1 & 61.3 & 38.2 & 0.0041 & -6.5 & -4.78 & II \\
\hline EN271015_220749 & SB & 33.75 & 94.3 & 71.6 & 62.4 & 41.9 & 0.20 & -10.5 & -5.52 & IIIA \\
\hline EN281015_011855 & S & 28.87 & 90.1 & 77.9 & 65.8 & 42.8 & 0.0023 & -5.0 & -4.98 & II \\
\hline EN301015_222401 & SB & 32.98 & 104.0 & 67.5 & 65.5 & 38.9 & 0.0047 & -8.9 & -5.04 & II \\
\hline EN311015_002325 & $\mathrm{N}$ & 30.38 & 102.7 & 68.1 & 59.3 & 32.0 & 0.0037 & -6.3 & -4.77 & II \\
\hline EN311015_023900 & SB & 32.63 & 105.5 & 80.9 & 68.5 & 47.5 & 0.0055 & -6.3 & -5.17 & II \\
\hline EN311015_025717 & SB & 32.63 & 99.8 & 71.7 & 57.9 & 53.3 & 0.21 & -11.5 & -5.19 & II \\
\hline EN311015_172431 & SB & 33.05 & 102.0 & 87.4 & 81.9 & 76.5 & 0.0010 & -3.5 & -5.12 & II \\
\hline EN311015_180520 & SB & 33.06 & 114.7 & 80.8 & 57.6 & 72.1 & 1300 & -18.6 & -6.31 & IIIB \\
\hline EN311015_182902 & SB & 32.61 & 99.0 & 79.0 & 74.0 & 69.8 & 0.0005 & -4.0 & -4.66 & II \\
\hline EN311015_185530 & SB & 32.80 & 103.7 & 77.1 & 69.4 & 63.7 & 0.0006 & -3.0 & -4.55 & II \\
\hline EN311015_192126 & $\mathrm{S}$ & 31.90 & 99.9 & 69.9 & 69.5 & 62.6 & 0.0006 & -3.5 & -4.62 & II \\
\hline EN311015_200534 & SB & 32.76 & 100.3 & 76.7 & 67.2 & 52.2 & 0.0020 & -5.1 & -4.85 & II \\
\hline EN311015_202117 & SB & 32.43 & 105.7 & 60.3 & 55.2 & 53.5 & 0.0070 & -8.0 & -4.43 & I \\
\hline EN311015_211904 & SB & 33.08 & 102.8 & 75.5 & 71.3 & 46.8 & 0.039 & -10.2 & -5.68 & IIIB \\
\hline EN311015_230919 & SB & 32.78 & 100.2 & 69.9 & 64.1 & 33.6 & 0.0006 & -3.8 & -4.61 & II \\
\hline EN311015_231301 & SB & 32.56 & 120.0 & 74.4 & 57.3 & 36.9 & 34 & -15.8 & -6.19 & IIIB \\
\hline EN011115_013625 & SB & 32.41 & 98.8 & 69.0 & 58.2 & 41.0 & 0.021 & -9.5 & -4.92 & II \\
\hline EN011115_033911 & $\mathrm{N}$ & 32.92 & 101.2 & 74.7 & 71.6 & 54.0 & 0.0081 & -9.4 & -5.33 & IIIA \\
\hline EN011115_174410 & SB & 32.64 & 102.3 & 71.7 & 67.2 & 75.6 & 0.0089 & -6.6 & -4.62 & II \\
\hline EN011115_183646 & $\mathrm{S}$ & 32.55 & 95.0 & 82.3 & 80.1 & 65.8 & 0.0002 & -2.4 & -5.00 & II \\
\hline EN011115_191104 & SB & 32.24 & 104.3 & 78.4 & 73.8 & 63.4 & 0.0049 & -4.1 & -5.00 & II \\
\hline EN011115_200918 & SB & 32.47 & 93.2 & 64.5 & 61.3 & 53.2 & 0.0030 & -6.9 & -4.60 & II \\
\hline EN011115_223909 & SB & 32.52 & 102.2 & 76.5 & 62.8 & 37.3 & 0.0021 & -4.6 & -4.72 & II \\
\hline EN011115_234207 & SB & 32.60 & 96.3 & 71.5 & 57.6 & 31.3 & 0.0083 & -7.5 & -4.77 & II \\
\hline EN021115_020950 & SB & 32.05 & 99.8 & 77.0 & 69.7 & 45.1 & 0.0020 & -6.1 & -5.09 & II \\
\hline EN021115_021740 & SB & 32.00 & 94.0 & 74.9 & 68.2 & 45.1 & 0.0008 & -4.2 & -4.83 & II \\
\hline EN021115_022525 & SB & 32.38 & 107.5 & 75.2 & 63.3 & 45.3 & 0.20 & -10.9 & -5.57 & IIIA \\
\hline EN021115_024553 & $\mathrm{N}$ & 29.72 & 99.9 & 59.3 & 53.9 & 47.5 & 0.027 & -7.8 & -4.68 & II \\
\hline EN021115_182450 & SB & 31.93 & 94.9 & 72.7 & 62.7 & 71.2 & 0.031 & -7.8 & -4.76 & II \\
\hline EN021115_195540 & SB & 32.46 & 100.8 & 79.0 & 75.1 & 55.8 & 0.0018 & -5.3 & -5.25 & IIIA \\
\hline EN021115_201534 & SB & 32.19 & 102.9 & 76.5 & 72.1 & 53.6 & 0.0002 & -2.1 & -4.73 & II \\
\hline EN021115_205431 & $\mathrm{N}$ & 33.00 & 111.9 & 76.5 & 73.8 & 42.9 & 0.0003 & -3.2 & -4.98 & II \\
\hline EN021115_213614 & SB & 32.17 & 97.5 & 70.8 & 60.7 & 45.4 & 0.15 & -11.3 & -5.37 & IIIA \\
\hline EN021115_215818 & SB & 32.16 & 99.5 & 77.0 & 73.3 & 40.8 & 0.0017 & -6.7 & -5.31 & IIIA \\
\hline EN021115_220435 & SB & 32.11 & 108.6 & 70.8 & 63.6 & 39.3 & 0.0010 & -6.1 & -4.68 & II \\
\hline EN021115_232112 & SB & 31.80 & 94.4 & 54.1 & 48.4 & 35.5 & 0.015 & -9.3 & -4.39 & I \\
\hline EN021115_234348 & $\mathrm{S}$ & 30.66 & 100.5 & 78.5 & 70.9 & 32.8 & 0.0001 & -1.9 & -4.78 & II \\
\hline EN021115_235259 & SB & 31.98 & 100.5 & 62.9 & 58.9 & 33.2 & 0.0031 & -7.5 & -4.68 & II \\
\hline EN031115_002007 & SB & 31.78 & 101.9 & 73.3 & 58.7 & 35.1 & 0.0004 & -3.0 & -4.28 & I \\
\hline EN031115_011247 & SB & 32.10 & 98.8 & 81.0 & 67.4 & 37.9 & 0.0003 & -2.6 & -4.67 & II \\
\hline EN031115_012404 & SB & 31.87 & 97.6 & 71.6 & 61.1 & 41.9 & 0.0015 & -4.3 & -4.59 & II \\
\hline EN031115_025102 & SB & 31.85 & 99.8 & 73.0 & 69.1 & 48.4 & 0.0004 & -3.0 & -4.71 & II \\
\hline EN031115_031920 & SB & 31.43 & 97.5 & 81.5 & 71.3 & 56.1 & 0.0005 & -3.2 & -4.80 & II \\
\hline EN031115_193751 & SB & 31.99 & 102.6 & 80.3 & 69.9 & 57.8 & 0.0053 & -6.4 & -5.12 & II \\
\hline EN031115_195654 & SB & 32.13 & 97.7 & 69.1 & 68.4 & 54.6 & 0.0055 & -9.1 & -5.09 & II \\
\hline EN031115_202247 & $\mathrm{N}$ & 32.12 & 94.4 & 79.1 & 72.8 & 44.5 & 0.0008 & -4.2 & -5.12 & II \\
\hline EN031115_204226 & SB & 31.84 & 100.9 & 65.9 & 59.2 & 51.0 & 0.0013 & -4.9 & -4.37 & I \\
\hline
\end{tabular}

Notes. The entry velocity, heights of beginning, maximum brightness and end, average zenith distance of the radiant, photometric mass, maximum absolute magnitude, PE coefficient, and classification according to PE are given. Code of each fireball contains also date (in ddmmyy format) and GMT time corresponding to beginning rounded to whole second (in hhmmss format). 
Table A.2. continued.

\begin{tabular}{|c|c|c|c|c|c|c|c|c|c|c|}
\hline Code & Branch & $\begin{array}{r}v_{\infty} \\
\mathrm{km} \mathrm{s}^{-1}\end{array}$ & $\begin{array}{r}H_{\text {beg }} \\
\mathrm{km}\end{array}$ & $\begin{array}{r}H_{\max } \\
\mathrm{km}\end{array}$ & $\begin{array}{r}H_{\text {end }} \\
\mathrm{km}\end{array}$ & $\begin{array}{l}Z_{\mathrm{rad}} \\
\text { deg }\end{array}$ & $\begin{array}{r}\text { Mass } \\
\text { kg }\end{array}$ & Mag & $\mathrm{PE}$ & Type \\
\hline EN031115_212219 & SB & 31.92 & 96.5 & 69.6 & 68.1 & 42.1 & 0.0017 & -6.7 & -4.99 & II \\
\hline EN031115_212455 & SB & 32.04 & 99.1 & 64.8 & 59.8 & 41.7 & 0.013 & -9.8 & -4.92 & II \\
\hline EN031115_213844 & SB & 31.77 & 101.5 & 72.7 & 66.8 & 42.9 & 0.0029 & -6.5 & -5.01 & II \\
\hline EN031115_221917 & SB & 31.63 & 101.6 & 83.8 & 74.6 & 38.5 & 0.0003 & -2.9 & -5.08 & II \\
\hline EN031115_221937 & SB & 31.82 & 98.9 & 64.6 & 59.9 & 39.6 & 0.0047 & -7.7 & -4.77 & II \\
\hline EN031115_222446 & SB & 31.19 & 100.8 & 72.9 & 69.4 & 36.2 & 0.0003 & -2.6 & -4.78 & II \\
\hline EN031115_225609 & SB & 32.06 & 95.9 & 72.0 & 61.7 & 35.7 & 0.0008 & -3.8 & -4.57 & I \\
\hline EN031115_230149 & SB & 31.78 & 99.1 & 74.5 & 66.1 & 33.1 & 0.0010 & -4.7 & -4.85 & II \\
\hline EN031115_232829 & SB & 31.85 & 98.6 & 69.5 & 59.2 & 34.9 & 0.0030 & -7.0 & -4.68 & II \\
\hline EN031115_235911 & $\mathrm{S}$ & 30.41 & 98.1 & 76.1 & 66.8 & 30.1 & 0.012 & -8.4 & -5.40 & IIIA \\
\hline EN041115_012728 & SB & 31.16 & 106.5 & 63.7 & 48.1 & 39.5 & 0.0018 & -4.1 & -3.97 & I \\
\hline EN041115_020201 & SB & 31.10 & 98.1 & 63.9 & 58.1 & 45.9 & 0.0033 & -6.3 & -4.56 & I \\
\hline EN041115_021111 & SB & 31.80 & 100.2 & 71.7 & 67.2 & 42.5 & 0.44 & -12.7 & -5.96 & IIIB \\
\hline EN041115_021452 & SB & 31.50 & 102.8 & 68.4 & 63.8 & 45.1 & 0.0076 & -8.8 & -5.01 & II \\
\hline EN041115_043317 & SB & 31.22 & 101.8 & 83.0 & 75.3 & 65.7 & 0.0005 & -3.0 & -4.88 & II \\
\hline EN041115_044559 & SB & 31.39 & 100.1 & 82.7 & 75.4 & 65.8 & 0.0005 & -2.6 & -4.89 & II \\
\hline EN041115_203853 & SB & 31.90 & 95.0 & 74.9 & 60.2 & 48.2 & 0.21 & -10.6 & -5.39 & IIIA \\
\hline EN041115_210403 & SB & 31.81 & 98.7 & 64.7 & 62.9 & 45.1 & 0.031 & -10.1 & -5.21 & II \\
\hline EN041115_214032 & SB & 31.58 & 94.1 & 68.2 & 56.2 & 38.3 & 0.016 & -9.8 & -4.81 & II \\
\hline EN041115_215226 & SB & 31.60 & 93.9 & 64.2 & 52.6 & 40.1 & 0.0032 & -4.2 & -4.29 & I \\
\hline EN041115_225243 & SB & 31.45 & 97.3 & 67.9 & 60.4 & 35.4 & 0.0015 & -5.6 & -4.61 & II \\
\hline EN041115_231355 & SB & 31.56 & 92.3 & 71.7 & 63.5 & 35.5 & 0.019 & -9.2 & -5.25 & IIIA \\
\hline EN051115_023102 & SB & 31.27 & 98.0 & 65.7 & 56.9 & 47.8 & 0.011 & -8.8 & -4.69 & II \\
\hline EN051115_183559 & $\mathrm{S}$ & 30.84 & 99.9 & 68.3 & 65.3 & 67.2 & 0.0087 & -7.8 & -4.80 & II \\
\hline EN051115_185259 & SB & 31.57 & 99.7 & 79.6 & 75.7 & 64.4 & 0.0004 & -3.3 & -4.88 & II \\
\hline EN051115_190203 & SB & 31.61 & 99.6 & 78.5 & 72.5 & 63.0 & 0.0008 & -3.6 & -4.85 & II \\
\hline EN051115_203651 & SB & 31.35 & 101.1 & 71.7 & 66.3 & 47.6 & 0.0010 & -6.3 & -4.76 & II \\
\hline EN051115_205304 & $\mathrm{N}$ & 31.46 & 103.5 & 70.0 & 57.4 & 38.5 & 0.093 & -10.4 & -5.20 & II \\
\hline EN051115_212802 & SB & 31.27 & 96.5 & 72.3 & 65.5 & 43.7 & 0.0016 & -4.7 & -4.84 & II \\
\hline EN051115_213128 & SB & 31.32 & 91.4 & 70.8 & 66.3 & 43.7 & 0.090 & -10.5 & -5.62 & IIIA \\
\hline EN051115_213433 & SB & 31.03 & 105.9 & 62.2 & 58.1 & 41.0 & 0.0038 & -7.4 & -4.63 & II \\
\hline EN051115_220108 & SB & 31.05 & 99.3 & 70.8 & 66.0 & 38.9 & 0.050 & -10.0 & -5.54 & IIIA \\
\hline EN051115_221253 & SB & 31.28 & 102.4 & 64.7 & 60.9 & 36.6 & 0.010 & -9.6 & -4.99 & II \\
\hline EN051115_221501 & $\mathrm{N}$ & 30.95 & 107.8 & 77.0 & 70.8 & 29.9 & 0.0005 & -3.9 & -5.02 & II \\
\hline EN051115_221906 & SB & 31.28 & 96.7 & 76.3 & 67.6 & 35.5 & 0.0006 & -4.0 & -4.84 & II \\
\hline EN051115_225625 & SB & 31.10 & 99.9 & 76.7 & 71.2 & 35.2 & 0.0003 & -3.1 & -4.90 & II \\
\hline EN051115_225852 & S & 30.66 & 98.7 & 73.3 & 63.7 & 34.9 & 0.0019 & -5.7 & -4.85 & II \\
\hline EN051115_231201 & SB & 31.22 & 105.3 & 72.0 & 62.1 & 32.3 & 0.11 & -11.0 & -5.52 & IIIA \\
\hline EN051115_232719 & $\mathrm{N}$ & 31.48 & 101.0 & 71.0 & 64.6 & 25.4 & 0.0018 & -6.2 & -4.94 & II \\
\hline EN051115_234939 & SB & 31.05 & 102.5 & 61.5 & 57.0 & 34.2 & 0.0026 & -6.5 & -4.56 & I \\
\hline EN051115_235119 & SB & 31.37 & 99.3 & 73.7 & 63.7 & 33.4 & 0.0019 & -6.8 & -4.85 & II \\
\hline EN061115_001740 & SB & 31.20 & 104.8 & 68.5 & 61.1 & 35.2 & 0.0044 & -7.8 & -4.86 & II \\
\hline EN061115_002202 & S & 30.20 & 102.2 & 62.9 & 54.0 & 33.4 & 0.0009 & -4.3 & -4.22 & I \\
\hline EN061115_003508 & SB & 31.10 & 100.4 & 66.6 & 65.3 & 36.5 & 0.0052 & -9.1 & -5.11 & II \\
\hline EN061115_005009 & $\mathrm{S}$ & 31.20 & 94.2 & 74.4 & 64.2 & 37.9 & 0.0045 & -7.8 & -5.01 & II \\
\hline EN061115_011233 & SB & 31.02 & 98.9 & 60.1 & 57.5 & 38.5 & 0.0031 & -8.1 & -4.58 & I \\
\hline EN061115_011441 & SB & 30.67 & 98.9 & 83.6 & 71.9 & 37.0 & 0.0003 & -3.4 & -4.94 & II \\
\hline EN061115_011623 & SB & 30.85 & 97.2 & 69.0 & 64.5 & 39.9 & 0.0004 & -2.9 & -4.57 & I \\
\hline EN061115_025156 & SB & 30.98 & 94.5 & 77.5 & 73.2 & 50.1 & 0.0026 & -5.0 & -5.31 & IIIA \\
\hline EN061115_030548 & $\mathrm{S}$ & 31.33 & 98.8 & 75.9 & 71.7 & 51.5 & 0.0020 & -7.3 & -5.15 & II \\
\hline EN061115_040629 & SB & 30.78 & 101.1 & 71.1 & 65.0 & 63.3 & 0.0100 & -8.2 & -4.89 & II \\
\hline EN061115_164758 & SB & 31.28 & 104.0 & 79.7 & 74.0 & 83.3 & 0.0098 & -5.1 & -4.64 & II \\
\hline EN061115_174311 & SB & 31.47 & 99.6 & 79.8 & 76.5 & 73.3 & 0.0018 & -4.2 & -4.99 & II \\
\hline EN071115_015331 & SB & 30.60 & 104.4 & 76.7 & 59.0 & 43.7 & 3.6 & -13.8 & -5.92 & IIIB \\
\hline EN081115_010613 & SB & 30.59 & 92.4 & 78.7 & 71.5 & 37.9 & 0.0014 & -5.9 & -5.23 & II \\
\hline EN081115_033341 & SB & 30.41 & 96.4 & 72.3 & 70.0 & 57.5 & 0.0017 & -5.3 & -4.95 & II \\
\hline EN081115_181258 & SB & 30.69 & 99.1 & 65.9 & 65.6 & 70.2 & 0.049 & -9.8 & -5.05 & II \\
\hline EN081115_202907 & SB & 30.35 & 100.2 & 68.1 & 66.6 & 50.0 & 0.0020 & -6.3 & -4.90 & II \\
\hline
\end{tabular}


P. Spurný et al.: Discovery of a new branch of the Taurid meteoroid stream

Table A.2. continued.

\begin{tabular}{|c|c|c|c|c|c|c|c|c|c|c|}
\hline Code & Branch & $\begin{array}{r}v_{\infty} \\
\mathrm{km} \mathrm{s}^{-1}\end{array}$ & $\begin{array}{r}H_{\text {beg }} \\
\mathrm{km}\end{array}$ & $\begin{array}{r}H_{\max } \\
\mathrm{km}\end{array}$ & $\begin{array}{r}H_{\text {end }} \\
\mathrm{km}\end{array}$ & $\begin{array}{l}Z_{\mathrm{rad}} \\
\text { deg }\end{array}$ & $\begin{array}{r}\text { Mass } \\
\mathrm{kg}\end{array}$ & Mag & $\mathrm{PE}$ & Type \\
\hline EN081115_212839 & SB & 30.08 & 103.1 & 68.3 & 57.6 & 41.3 & 0.0022 & -5.0 & -4.52 & I \\
\hline EN081115_234417 & SB & 30.10 & 99.4 & 70.9 & 63.4 & 33.0 & 0.0062 & -7.9 & -5.08 & II \\
\hline EN091115_001801 & SB & 29.90 & 102.9 & 77.6 & 72.4 & 33.3 & 0.0009 & -5.2 & -5.25 & II \\
\hline EN091115_003545 & SB & 29.97 & 96.2 & 69.0 & 53.2 & 33.5 & 0.041 & -9.3 & -4.85 & II \\
\hline EN091115_011246 & SB & 29.73 & 88.7 & 75.7 & 70.3 & 40.5 & 0.0015 & -5.0 & -5.15 & II \\
\hline EN091115_011650 & SB & 29.88 & 98.4 & 76.5 & 71.4 & 38.5 & 0.0003 & -3.3 & -4.96 & II \\
\hline EN091115_032502 & SB & 30.13 & 99.6 & 78.8 & 73.4 & 54.7 & 0.0015 & -4.5 & -5.18 & IIIA \\
\hline EN091115_041944 & $\mathrm{N}$ & 31.62 & 99.3 & 73.9 & 67.8 & 61.1 & 0.0052 & -8.5 & -4.95 & II \\
\hline EN101115_212402 & SB & 29.77 & 102.4 & 63.5 & 58.4 & 41.5 & 0.011 & -9.6 & -4.86 & II \\
\hline EN101115_235401 & SB & 29.75 & 98.1 & 71.7 & 66.3 & 33.5 & 0.12 & -10.9 & -5.78 & IIIB \\
\hline EN111115_004713 & $\mathrm{S}$ & 29.11 & 97.3 & 63.9 & 56.9 & 35.8 & 0.022 & -9.6 & -4.97 & II \\
\hline EN111115_031037 & SB & 29.55 & 95.0 & 71.6 & 65.9 & 54.8 & 0.91 & -13.5 & -5.92 & IIIB \\
\hline EN111115_181413 & SB & 29.76 & 97.2 & 72.0 & 62.1 & 66.6 & 0.23 & -11.4 & -5.26 & IIIA \\
\hline EN111115_181509 & SB & 29.66 & 98.5 & 74.1 & 69.2 & 65.3 & 0.27 & -11.8 & -5.70 & IIIB \\
\hline EN111115_184540 & $\mathrm{N}$ & 31.36 & 102.0 & 65.9 & 72.0 & 59.4 & 0.0010 & -3.3 & -4.92 & II \\
\hline EN111115_203917 & SB & 29.47 & 93.5 & 77.6 & 74.5 & 46.5 & 0.0010 & -3.8 & -5.29 & IIIA \\
\hline EN111115_233243 & SB & 29.51 & 98.9 & 68.1 & 60.1 & 32.4 & 0.014 & -8.3 & -5.07 & II \\
\hline EN121115_004717 & SB & 29.07 & 96.9 & 74.4 & 71.6 & 36.9 & 0.0008 & -5.4 & -5.17 & II \\
\hline EN121115_232341 & $\mathrm{S}$ & 28.20 & 95.7 & 74.2 & 66.6 & 33.8 & 0.0005 & -3.4 & -4.83 & II \\
\hline EN131115_002058 & SB & 29.48 & 97.4 & 77.4 & 66.1 & 36.0 & 0.0074 & -8.2 & -5.25 & IIIA \\
\hline EN131115_004858 & SB & 29.34 & 94.5 & 79.2 & 67.7 & 35.8 & 0.0003 & -2.3 & -4.76 & II \\
\hline EN131115_015008 & SB & 29.17 & 103.1 & 74.7 & 61.2 & 43.8 & 0.0014 & -5.0 & -4.63 & II \\
\hline EN131115_042559 & SB & 28.90 & 93.7 & 75.9 & 74.2 & 64.1 & 0.0009 & -3.5 & -5.01 & II \\
\hline EN161115_193458 & $\mathrm{S}$ & 28.19 & 101.5 & 60.8 & 56.0 & 53.5 & 0.0079 & -6.1 & -4.58 & I \\
\hline EN161115_213048 & SB & 28.28 & 100.4 & 70.5 & 59.4 & 39.6 & 0.017 & -10.1 & -5.05 & II \\
\hline EN161115_222246 & $\mathrm{S}$ & 31.25 & 97.9 & 74.3 & 69.1 & 35.2 & 0.0004 & -3.6 & -4.86 & II \\
\hline EN171115_020907 & SB & 28.26 & 93.5 & 68.8 & 62.0 & 46.6 & 0.0062 & -6.2 & -4.93 & II \\
\hline EN171115_022102 & SB & 27.98 & 95.1 & 66.2 & 59.0 & 47.5 & 0.049 & -10.6 & -5.16 & II \\
\hline EN231115_012005 & $\mathrm{N}$ & 28.37 & 93.2 & 64.7 & 57.5 & 33.2 & 0.0040 & -5.8 & -4.72 & II \\
\hline EN231115_224311 & S & 28.06 & 96.8 & 68.5 & 57.0 & 31.7 & 0.074 & -10.3 & -5.25 & II \\
\hline EN281115_195251 & S & 27.74 & 108.0 & 78.2 & 64.7 & 50.3 & 0.0016 & -4.1 & -4.80 & II \\
\hline
\end{tabular}

